\title{
Perfectly invisible $\mathcal{P} \mathcal{T}$-symmetric zero-gap systems, conformal field theoretical kinks, and exotic nonlinear supersymmetry
}

\author{
Juan Mateos Guilarte ${ }^{a}$ and Mikhail S. Plyushchay ${ }^{b}$ \\ ${ }^{a}$ Departamento de Física Fundamental and IUFFyM, Universidad de Salamanca, \\ Casas del Parque II, Salamanca E-37008, Spain \\ ${ }^{b}$ Departamento de Física, Universidad de Santiago de Chile, \\ Casilla 30\%, Santiago 2, Chile \\ E-mail: guilarte@usal.es, mikhail.plyushchay@usach.cl
}

Abstract: We investigate a special class of the $\mathcal{P} \mathcal{T}$-symmetric quantum models being perfectly invisible zero-gap systems with a unique bound state at the very edge of continuous spectrum of scattering states. The family includes the $\mathcal{P} \mathcal{T}$-regularized two particle Calogero systems (conformal quantum mechanics models of de Alfaro-Fubini-Furlan) and their rational extensions whose potentials satisfy equations of the KdV hierarchy and exhibit, particularly, a behaviour typical for extreme waves. We show that the two simplest Hamiltonians from the Calogero subfamily determine the fluctuation spectra around the $\mathcal{P} \mathcal{T}$-regularized kinks arising as traveling waves in the field-theoretical Liouville and SU(3) conformal Toda systems. Peculiar properties of the quantum systems are reflected in the associated exotic nonlinear supersymmetry in the unbroken or partially broken phases. The conventional $\mathcal{N}=2$ supersymmetry is extended here to the $\mathcal{N}=4$ nonlinear supersymmetry that involves two bosonic generators composed from Lax-Novikov integrals of the subsystems, one of which is the central charge of the superalgebra. Jordan states are shown to play an essential role in the construction.

Keywords: Conformal and W Symmetry, Discrete Symmetries, Extended Supersymmetry, Integrable Hierarchies

ARXIV EPRINT: 1710.00356 


\section{Contents}

1 Introduction $\quad 1$

2 Perfectly invisible $\mathcal{P} \mathcal{T}$-symmetric zero-gap systems $\quad 3$

$2.1 \mathcal{P} \mathcal{T}$-regularized Calogero systems 3

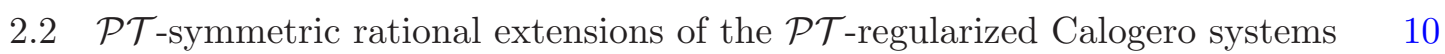

3 (1+1)-dimensional conformal field theoretical kinks seen from conformal quantum mechanics

3.1 Kinks in Liouville field theory 16

$\begin{array}{lll}3.2 \text { Kinks in the SU(3) conformal Toda model } & 19\end{array}$

4 Exotic supersymmetry of the pairs of perfectly invisible $\mathcal{P} \mathcal{T}$-symmetric systems

5 Summary, discussion and outllok

A Quantum scattering on the half-line

\section{Introduction}

$\mathcal{P} \mathcal{T}$-symmetric quantum systems [1] have many interesting properties and attract a lot of attention, for reviews see $[2,3]$. Some time ago there was revealed their close relationship with certain two-dimensional integrable quantum field theories in the context of the ODE/IM correspondence $[4,5]$. On the other hand, many works were devoted to investigation of diverse aspects of the $\mathcal{P} \mathcal{T}$-symmetric regularizations, extensions and deformations of Calogero systems [6-13]. Calogero systems, as is well known, govern the dynamics of the moving poles of rational solutions to the Korteweg-de Vries (KdV) equation [14-16]. $\mathcal{P} \mathcal{T}$-symmetric quantum systems also reveal peculiar properties from the perspective of supersymmetric quantum mechanics [17-22].

Solitonic (reflectionless) and finite-gap periodic quantum systems and their rational, singular on real line, limit cases are intimately related to the KdV equation via the inverse scattering transform. The KdV equation (as well as equations of the KdV hierarchy) appears as a compatibility condition of the overdetermined system of two equations imposed on one wave function. One of them has a form of the stationary quantum Schrödinger equation with a spectral parameter. Another defines the evolution of the wave function in a time variable on which potential depends as on external parameter corresponding to an isospectral deformation of the Schrödinger equation. Such reformulation of the KdV equation, introduced by Lax, naturally explains the appearance of the inverse scattering transform 
under construction of soliton solutions. The Lax representation also plays a fundamental role in algebro-geometric method developed for the construction of finite-gap (finite-zone) periodic and quasi-periodic solutions [23]. The overdertermined system of equations in the Lax representation is covariant under Darboux transformations. This covariance allows to employ the Darboux transformations as alternative method for construction of both stationary and time-dependent solutions to the KdV equation and equations of the hierarchy [24]. Darboux transformations can also be used for construction of time-dependent self-similar rational solutions to the KdV equation [25, 26]. The corresponding potentials in the last case are of the type of potentials of the Calogero model being singular functions with a double pole on the real line. Any solution of the KdV equation satisfies the ordinary differential equation known as Novikov equation which is a higher stationary KdV equation [23]. From the viewpoint of the associated Schrödinger operator, such higher stationary KdV equation can be reinterpreted in terms of a nontrivial Lax-Novikov integral. It is this higher order differential operator that detects all the bound states in the associated Schrödinger quantum system with a soliton potential, or the edge-states of the valence and conduction bands in the spectrum of a system with periodic finite-gap potential, and both bound and edge states in the solutions to the $\mathrm{KdV}$ equation with solitons in a stationary asymptotically periodic background [27]. This operator also separates the deformed plane waves of opposite chirality in the reflectionless systems with soliton potentials, and Bloch states with opposite values of quasi-momentum inside the valence and conduction bands in finite-gap systems. The Lax-Novikov higher order differential operator also exists in any quantum system with the KdV rational potential of the Calogero type. However, in rational case it is a formally commuting with Hamiltonian operator which has a non-physical nature due to a singularity of the potential: acting on physical quantum states which nullify at the potential's pole, it transforms them into non-physical wave functions singular at the position of the pole [28].

In the present paper we construct and investigate a special class of the perfectly invisible $\mathcal{P} \mathcal{T}$-symmetric zero-gap quantum mechanical systems related to the $\mathrm{KdV}$ hierarchy. They represent the $\mathcal{P} \mathcal{T}$-regularized two-particle Calogero systems (conformal quantum mechanics models of de Alfaro-Fubini-Furlan [29]) and their rational extensions, which have the unique bound state at the very edge of the continuous spectrum of scattering states, and are characterized by transmission amplitude equal to one.

The $\mathcal{P} \mathcal{T}$-symmetric regularization is achieved here via a simple imaginary shift of the coordinate, $x \rightarrow x+i \alpha, \alpha \in \mathbb{R} .^{1}$ As a result, the Lax-Novikov operator will transform into a true integral of the corresponding quantum system. The real and imaginary parts of the potential with 'reconstructed' time-dependence will provide us with interesting solutions to the KdV and higher equations of the hierarchy which exhibit, particularly, the behaviour typical for the extreme (rogue) waves. The corresponding potentials are constructed via Darboux transformations which use as the seed states a complex non-physical eigenstate of zero energy of a free quantum particle and the Jordan states related to it. As a consequence, each of the obtained quantum systems will possess a unique bound state of zero energy at

\footnotetext{
${ }^{1}$ For $\mathcal{P} \mathcal{T}$-symmetric deformations of the $\mathrm{KdV}$ and other integrable systems in spirit of [1], see refs. [30, 31] and $[32,33]$.
} 
the very edge of the continuous part of the spectrum. Since the quantum $\mathcal{P} \mathcal{T}$-symmetric systems are generated from the free particle, and their potentials are non-singular on the real line, the obtained systems will be reflectionless. In the case of usual reflectionless systems with soliton potential, the transmission amplitude is a pure phase which is a rational function of energy of scattering states with zeroes and poles defined by energies of the bound states. In the quantum $\mathcal{P} \mathcal{T}$-symmetric systems we study here, there is a unique bound state of zero energy that is located at the very edge of the continuous part of the spectrum. As a consequence, the transmission amplitude does not depend on energy of the scattering states and reduces to a constant value equal to 1 . This means that the systems we consider are not just reflectionless, but are perfectly invisible systems. Such quantum systems are studied, particularly, in the context of quantum optics [34-36]. The described peculiarities of the perfectly invisible zero-gap quantum systems lead to unusual properties of the corresponding supersymmetrically extended systems. Because of the presence of the nontrivial Lax-Novikov integrals of motion, instead of the conventional $\mathcal{N}=2$ supersymmetry, the Darboux-related quantum pairs will be described by exotic $\mathcal{N}=4$ nonlinear supersymmetry. The anti-commutators of linear and higher order supercharges appearing here generate the Lax-Novikov integral of the extended system, which plays a role of the bosonic central charge of the superalgebra. We also will show that the two simplest Hamiltonians from the Calogero subfamily determine the fluctuation spectra around the $\mathcal{P} \mathcal{T}$-regularized kinks arising as traveling waves in the field-theoretical Liouville and SU(3) conformal Toda systems.

The paper is organized as follows. In the next section 2 we construct the indicated class of the quantum systems by applying the appropriate Darboux-Crum transformations to a free particle. We investigate their relationship with the stationary and non-stationary equations of the KdV hierarchy, and describe the properties of the higher-derivative LaxNovikov integrals in such systems. We also consider there some particular time-dependent $\mathcal{P} \mathcal{T}$-symmetric potentials whose real and imaginary parts have a soliton-like form with a behaviour typical for extreme waves. In section 3 we study $(1+1)$-dimensional conformal field theoretical kinks appearing in the Liouville and SU(3) conformal Toda systems and establish their relation with two simplest cases of perfectly invisible $\mathcal{P} \mathcal{T}$-symmetric Calogero systems. In section 4 we discuss exotic $\mathcal{N}=4$ nonlinear supersymmetry of the extended systems composed from the pairs of $\mathcal{P} \mathcal{T}$-symmetric zero-gap systems related by the first order Darboux transformations. Section 5 is devoted to the summary, discussion and outlook. In appendix we briefly discuss a quantum scattering problem on the half-line.

\section{$2 \quad$ Perfectly invisible $\mathcal{P} \mathcal{T}$-symmetric zero-gap systems}

\section{$2.1 \quad \mathcal{P} \mathcal{T}$-regularized Calogero systems}

Consider a quantum free particle on $\mathbb{R}^{1}$ described by the Hamiltonian $H_{0}=-\frac{d^{2}}{d x^{2}}$. Eigenstates of $H_{0}$ are the plane waves $\psi_{0, \pm k}=e^{ \pm i k x}, k>0$, and any positive energy value $E_{k}=k^{2}>0$ is doubly degenerate. A non-degenerate eigenstate $\psi_{0,0}(x)=1$ of zero energy corresponds to a limit $k \rightarrow 0$ case of the plane waves $\psi_{0, \pm k}$. Like $\psi_{0, \pm k}, \psi_{0,0}$ is a bounded function on $\mathbb{R}^{1}$. A linear independent from $\psi_{0,0}(x)$ eigenstate of $H_{0}$ of zero eigenvalue is 
$\widetilde{\psi_{0,0}}=x$, that is a non-physical state unbounded at infinity. In general, a linear independent from $\psi(x)$ solution to a stationary Schrödinger equation $H \psi=E \psi$ can be taken in the form

$$
\widetilde{\psi(x)}=\psi(x) \int \frac{d \xi}{(\psi(\xi))^{2}} .
$$

Normalization in $(2.1)$ corresponds to the Wronskian value $W(\psi, \widetilde{\psi})=1$. The state $\widetilde{\psi_{0,0}}=x$ also can be obtained from the odd linear combination of the plane wave states of energy $k^{2}$ in a limit $k \rightarrow 0: \lim _{k \rightarrow 0}\left(\psi_{0,+k}-\psi_{0,-k}\right) / 2 i k=\widetilde{\psi_{0,0}}$.

The Hamiltonian operator $H_{0}$ has an integral of motion $\mathcal{P}_{0}=-i \frac{d}{d x},\left[H_{0}, \mathcal{P}_{0}\right]=0$. It distinguishes the left- and right-moving plane wave states inside the energy doublets, $\mathcal{P}_{0} \psi_{0, \pm k}= \pm k \psi_{0, \pm k}$. The state $\psi_{0,0}=1$ constitutes the kernel of $\mathcal{P}_{0}, \mathcal{P}_{0} \psi_{0,0}=0$, while nonphysical zero energy eigenstate $\widetilde{\psi_{0,0}}$ is transformed by $\mathcal{P}_{0}$ into the eigenstate $\psi_{0,0}, i \mathcal{P}_{0} x=1$. As $\left(\mathcal{P}_{0}\right)^{2} \widetilde{\psi_{0,0}}=0$, the state $\widetilde{\psi_{0,0}}$ is a Jordan state of $\mathcal{P}_{0}$ of order 2 [37].

Within a framework of Darboux transformations, one can construct the annihilator of the non-physical zero energy state $\widetilde{\psi_{0,0}}(x)=x: D_{1}=x \frac{d}{d x} x^{-1}=\frac{d}{d x}-\frac{1}{x}, D_{1} x=0$. The conjugate operator is $D_{1}^{\dagger}=-x^{-1} \frac{d}{d x} x=-\frac{d}{d x}-\frac{1}{x}$, and these two operators provide a factoirzation of $H_{0}$ :

$$
H_{0}=D_{1}^{\dagger} D_{1}=H_{0} .
$$

Their permuted product generates a conformal quantum mechanics model [29] given by a Hamiltonian for relative motion of a 2-particle Calogero system

$$
H_{1}=D_{1} D_{1}^{\dagger}=-\frac{d}{d x^{2}}+\frac{2}{x^{2}}
$$

with a value $g=2$ of the coupling constant. Unlike $H_{0}$, Hamiltonian $H_{1}$ is singular at $x=0$. If $H_{1}$ is considered on a whole real line punctured at the origin, the potential barrier at $x=0$ is not penetrable and the states with supports on $x<0$ and $x>0$ do not mix dynamically. The singularity of operators $H_{1}, D_{1}$ and $D_{1}^{\dagger}$ is associated with a node at $x=0$ of the non-physical zero energy eigenstate $\widetilde{\psi_{0,0}}(x)=x$ of $H_{0}$.

Potential $u_{1}=\frac{2}{x^{2}}$ in (2.3) is a stationary, singular on $\mathbb{R}^{1}$ solution [14] to the $\mathrm{KdV}$ equation

$$
u_{t}-6 u u_{x}+u_{x x x}=0 .
$$

It can be obtained from the one-soliton KdV solution

$$
u_{1}(x, t)=-\frac{2 \kappa^{2}}{\cosh ^{2} \kappa\left(x-4 \kappa^{2} t-\tau\right)}
$$

by fixing the soliton centre coordinate to be pure imaginary and equal $\tau=i \frac{\pi}{2 \kappa}$, and then by taking a limit $\kappa \rightarrow 0$. The limit $\kappa \rightarrow 0$ eliminates a length scale which controls the size of the one-soliton solution correlated with its velocity. As a result, the stationary Schrödinger equation $H_{1} \psi=E \psi$ is invariant under the transformation $x \rightarrow \alpha x, E \rightarrow \alpha^{-2} E$, that corresponds to a scale invariance of the system (2.3) similarly to the case of the free particle. A time-dependent solution $u_{1}(x, t)=-\frac{1}{6} c+\frac{2}{(x-c t)^{2}}$ to the KdV equation is obtained from the stationary solution $u_{1}(x)=\frac{2}{x^{2}}$ by employing the invariance of (2.4) under Galilean transformations, $u(x) \rightarrow u(x, t)=-\frac{1}{6} c+u(x-c t)$. It is a singular solution with a moving along $\mathbb{R}^{1}$ 
second order pole whose velocity $c$ is correlated with its background asymptotic value $-\frac{1}{6} c$. The time-dependent solution $u_{1}(x, t)$ also can be obtained directly from the one-soliton solution (2.5) in a more tricky way without making use of the Galilean symmetry. For this, after fixing $\tau=i \frac{\pi}{2 \kappa}$, in the resulting intermediate singular one-soliton solution $u=\frac{2 \kappa^{2}}{\sinh ^{2} \kappa X}$ to the $\mathrm{KdV}$ equation we take a limit $\kappa \rightarrow 0$ by preserving quadratic in $\kappa^{2}$ terms but leaving the composed argument $X=x-4 \kappa^{2} t$ untouched. In such a way we obtain $u=\frac{2}{X^{2}}-\frac{2}{3} \kappa^{2}$. Denoting then $4 \kappa^{2}=c$, we arrive at the same time-dependent singular solution $u_{1}(x, t)$.

Let us return to the Hamiltonian operators (2.2) and (2.3). From their factorization properties it follows that the operators $D_{1}$ and $D_{1}^{\dagger}$ intertwine $H_{0}$ and $H_{1}: D_{1} H_{0}=H_{1} D_{1}$, $H_{0} D_{1}^{\dagger}=D_{1}^{\dagger} H_{1}$. As a consequence, non-singular at $x=0^{+}$physical states of $H_{1}$ on half-line $x>0$ are obtained from the odd linear combination of the plane wave eigenfunctions of $H_{0}$, $\psi_{1, k}(x)=D_{1} \sin k x$. They satisfy the relations $H_{1} \psi_{1, k}=k^{2} \psi_{1, k}$ and $\lim _{x \rightarrow 0} \psi_{1, k}(x)=0$. The operator

$$
\mathcal{P}_{1}=D_{1} \mathcal{P}_{0} D_{1}^{\dagger}=i\left(\frac{d^{3}}{d x^{3}}-\frac{3}{x^{2}} \cdot \frac{d}{d x}+\frac{3}{x^{3}}\right),
$$

being a Darboux-dressed free particle integral $\mathcal{P}_{0}$, commutes with $H_{1}$. This is a LaxNovikov operator for the two-particle Calogero system (2.3). It is not, however, a true integral of motion of $H_{1}$ since acting on physical eigenstates $\psi_{1, k}(x)$ it transforms them into non-physical eigenstates of $H_{1}$ of the same energy $E=k^{2}>0$ which are singular at $x=0: \mathcal{P}_{1} \psi_{1, k}(x)=-i k^{3} D_{1} \cos k x=i k^{3}\left(k \sin k x+\frac{1}{x} \cos k x\right)$.

The singularity at $x=0$ of the intertwining operators $D_{1}$ and $D_{1}^{\dagger}$ and consequently of the Hamiltonian $H_{1}$ and operator $\mathcal{P}_{1}$ can be removed by a ' $\mathcal{P} \mathcal{T}$-regularization'. This is achieved by taking a complex linear combination of non-physical and physical zero energy eigenstates of $H_{0}$,

$$
\widetilde{\psi_{0,0}^{\alpha}}=x+i \alpha \equiv \xi
$$

where $\alpha$ is a nonzero real constant which, for definiteness, will be taken positive, $\alpha>0$. With the help of this state we construct the first order differential operators

$$
D_{1}=\xi \frac{d}{d x} \frac{1}{\xi}=\frac{d}{d x}-\xi^{-1}, \quad D_{1}^{\#}=-\frac{1}{\xi} \frac{d}{d x} \xi=-\frac{d}{d x}-\xi^{-1}
$$

whose kernels are, respectively, $\xi$ and $\xi^{-1}$. As before we have $H_{0}=D_{1}^{\#} D_{1}=-\frac{d^{2}}{d x^{2}}$. But now the partner Hamiltonian operator $H_{1}=D_{1} D_{1}^{\#}=-\frac{d^{2}}{d x^{2}}+2 \xi^{-2}$ is non-singular on the real line $\mathbb{R}^{1}$,

$$
H_{1}^{\alpha}=-\frac{d^{2}}{d x^{2}}+\frac{2}{(x+i \alpha)^{2}}
$$

and the first order differential operators (2.8) intertwine $H_{0}$ and $H_{1}^{\alpha}$,

$$
D_{1} H_{0}=H_{1}^{\alpha} D_{1}, \quad D_{1}^{\#} H_{1}^{\alpha}=H_{0} D_{1}^{\#} .
$$

Potential of the system (2.9) is obtained from the one-soliton solution (2.5) to the KdV equation if in the procedure described above the soliton centre parameter is fixed in the form $\tau=\frac{i}{\kappa}\left(\frac{\pi}{2}-\alpha\right)$. 
Hamiltonian (2.9) with a complex potential

$$
V_{1}(x)=2 \frac{x^{2}-\alpha^{2}}{\left(x^{2}+\alpha^{2}\right)^{2}}-4 i \alpha \frac{x}{\left(x^{2}+\alpha^{2}\right)^{2}}
$$

is $\mathcal{P} \mathcal{T}$-symmetric, $\left[H_{1}^{\alpha}, P T\right]=0$. Here $P$ is a space reflection operator, $P x=-x P, P^{2}=1$, and a complex conjugation operator $T$ is defined by $T z=\bar{z} T, T^{2}=1$, where $z \in \mathbb{C}$ is an arbitrary complex number. If we extend the definition of time reflection operator $T$ by a requirement $T t=-t T$, the time-dependent $\mathrm{KdV}$ equation (2.4) will be invariant under the $\mathcal{P} \mathcal{T}$ transformation if $u(x, t)$ is $\mathcal{P} \mathcal{T}$-symmetric: $[u(x, t), P T]=0$. Then the change $x \rightarrow X=x-c t$ accompanied by a shift for $-\frac{1}{6} c$ transforms potential (2.11) being stationary solution of the $\mathrm{KdV}$ equation into $\mathcal{P} \mathcal{T}$-symmetric time-dependent travelling wave solution $u(x, t)=V_{1}(X)-\frac{1}{6} c$ of the same equation.

The bounded on real line $\mathbb{R}$ eigenstates $\psi_{1, \pm k}^{\alpha}(x)=D_{1} e^{ \pm i k \xi}, k>0, H_{1}^{\alpha} \psi_{1, \pm k}^{\alpha}=k^{2} \psi_{1, \pm k}^{\alpha}$, can be considered as physical states of the $\mathcal{P} \mathcal{T}$-symmetric system (2.9). They are the Darboux-transformed plane wave eigenstates of $H_{0}$ that are obtained by applying to them the intertwining operator $D_{1}$ defined in (2.8). Unlike $H_{0}$, the system (2.9) has a gapless bound state of zero energy that lies at the very edge of the doubly degenerate continuous part of the spectrum. This square-integrable on $\mathbb{R}^{1}$ state can be obtained from the bounded but not square-integrable singlet zero energy eigenstate $\psi_{0,0}=1$ of $H_{0}$ by applying to the latter the intertwining operator $D_{1}:-D_{1} \psi_{0,0}=\xi^{-1} \equiv \psi_{1,0}^{\alpha}(x), H_{1}^{\alpha} \psi_{1,0}^{\alpha}=0$. The ground state $\xi^{-1}$ corresponds to a limit $k \rightarrow 0$ of the physical states $\psi_{1, \pm k}^{\alpha}$ from the continuous part of the spectrum, $\lim _{k \rightarrow 0}\left(-\psi_{1, \pm k}^{\alpha}\right)=\xi^{-1}$. It also can be obtained from the bound eigenstate $\kappa / \cosh \kappa(x+\tau)$ of eigenavalue $-\kappa^{2}$ of the reflectionless Pöschl-Teller system given by potential (2.5) at $t=0$ after fixing $\tau=\frac{i}{\kappa}\left(\frac{\pi}{2}-\alpha\right)$ and taking a limit $\kappa \rightarrow 0$. In this limit the energy gap separating the bound state from the continuous part of the spectrum disappears, and the bound eigenstate of the Pöschl-Teller system with one-soliton potential (2.5) transforms into the bound state of the system $H_{1}^{\alpha}$.

The peculiarity of the reflectionless $\mathcal{P} \mathcal{T}$-symmetric system (2.9) in comparison with Hermitian reflectionless systems is that the square-integrable on $\mathbb{R}^{1}$ singlet state here is not separated by a gap from the continuous doubly degenerate part of the spectrum with $E=k^{2}>0$. In this aspect it is similar to the periodic $\mathcal{P} \mathcal{T}$-symmetric finite-gap systems considered in [22]. It is also worth to emphasize here that the translational non-invariance of the $\mathcal{P} \mathcal{T}$-symmetric system (2.9) generated from the original translation-invariant free particle system $H_{0}$ is rooted in translational non-invariance of the zero energy eigenstate (2.7) of $H_{0}$ underlying the Darboux transformation.

Unlike (2.3), the $\mathcal{P} \mathcal{T}$-symmetric system (2.9) possesses a true integral of motion being a Darboux-dressed integral $\mathcal{P}_{0}$ of the free particle, $\mathcal{P}_{1}^{\alpha}=D_{1} \mathcal{P}_{0} D_{1}^{\#},\left[\mathcal{P}_{1}^{\alpha}, H_{1}^{\alpha}\right]=0$. This integral distinguishes the doublet states in the continuous part of the spectrum, $\mathcal{P}_{1}^{\alpha} \psi_{1, \pm k}^{\alpha}=$ $\pm k^{3} \psi_{1, \pm k}^{\alpha}$, and annihilates the ground state $\psi_{1,0}^{\alpha}=\xi^{-1}, \mathcal{P}_{1}^{\alpha} \psi_{1,0}^{\alpha}=0$. The complete kernel of the third order differential operator $\mathcal{P}_{1}^{\alpha}$ is

$$
\operatorname{ker} \mathcal{P}_{1}^{\alpha}=\operatorname{span}\left\{\xi^{-1}, \xi, \xi^{3}\right\} .
$$


It includes non-physical (undbounded) states $\xi$ and $\xi^{3}$. These two states are not eigenstates of $H_{1}^{\alpha}$, but they belong to the kernels of the operators $\left(H_{1}^{\alpha}\right)^{2}$ and $\left(H_{1}^{\alpha}\right)^{3}$, respectively:

$$
H_{1}^{\alpha} \xi^{3}=-4 \xi, \quad\left(H_{1}^{\alpha}\right) \xi=2 \xi^{-1}, \quad\left(H_{1}^{\alpha}\right)^{2} \xi=0, \quad\left(H_{1}^{\alpha}\right)^{3} \xi^{3}=0 .
$$

Thus the states $\xi$ and $\xi^{3}$ are the Jordan states of $H_{1}^{\alpha}$ of orders 2 and 3. In correspondence with (2.12) and (2.13), the operator $\mathcal{P}_{1}^{\alpha}$ satisfies a supersymmetry-like relation [38]

$$
\left(\mathcal{P}_{1}^{\alpha}\right)^{2}=\left(H_{1}^{\alpha}\right)^{3}
$$

concordant with the Burchnall-Chaundy theorem [39-42]. It is a Lax-Novikov integral for the finite-gap (zero-gap) $\mathcal{P} \mathcal{T}$-symmetric system $H_{1}^{\alpha}$.

A partner of the zero energy ground state $\xi^{-1}$ of the system $H_{1}^{\alpha}$ given by relation (2.1) is an unbounded state $\xi^{2}, H_{1}^{\alpha} \xi^{2}=0$. It does not belong to the kernel (2.12) like the non-physical zero energy eigenstate $\widetilde{\psi_{0,0}^{\alpha}}=\xi$ of $H_{0}$ does not belong to the kernel of the integral $\mathcal{P}_{0}$. The state $\xi^{2}$ is obtainable from the appropriate linear combination of the doublet states from the continuous part of the spectrum of $H_{1}^{\alpha}$ in a limit $k \rightarrow 0$ :

$$
\lim _{k \rightarrow 0} \frac{-3}{2 i k^{3}}\left(\psi_{1, k}^{\alpha}-\psi_{1,-k}^{\alpha}\right)=\xi^{2} .
$$

The operator $\mathcal{P}_{1}^{\alpha}$ transforms this non-physical zero energy state into a physical ground state, $\mathcal{P}_{1}^{\alpha} \xi^{2} \propto \xi^{-1}$, that can be compared with a similar picture in the case of the free particle system.

With respect to the Darboux transformation generator $D_{1}$, the pre-images of the states from the kernel of $\mathcal{P}_{1}$ are $1, \xi^{2}$ and $\xi^{4}: D_{1} 1=-\xi^{-1}, D_{1} \xi^{2}=\xi, D_{1} \xi^{4}=3 \xi^{3}$. Unlike the zero energy eigenstate $\psi_{0,0}=1$ of $H_{0}$, other two states satisfy the relations $H_{0} \xi^{4}=-12 \xi^{2}$ and $H_{0} \xi^{2}=-2$, i.e. $\xi^{2}$ and $\xi^{4}$ are the Jordan states of $H_{0}$ of orders 2 and 3, respectively. The nature of these three states related to the system $H_{0}$ is similar to that of their Darbouxcounterparts (2.12) in the system $H_{1}^{\alpha}$, cf. eq. (2.13).

The family of the systems

$$
H_{n}^{\alpha}=-\frac{d^{2}}{d x^{2}}+\frac{n(n+1)}{(x+i \alpha)^{2}}
$$

characterized by the integer parameter $n \geq 0$ is a hierarchy of the $\mathcal{P} \mathcal{T}$-symmetric reflectionless systems that includes the free particle $H_{0}$ and the system (2.9) as particular cases corresponding to the values $n=0$ and $n=1$. For neighbour members of the hierarchy, there are the factorization,

$$
H_{n-1}^{\alpha}=D_{n}^{\#} D_{n}, \quad H_{n}^{\alpha}=D_{n} D_{n}^{\#},
$$

and the intertwining,

$$
D_{n} H_{n-1}^{\alpha}=H_{n}^{\alpha} D_{n}, \quad D_{n}^{\#} H_{n}^{\alpha}=H_{n-1}^{\alpha} D_{n}^{\#},
$$

relations. Here the first order operators

$$
D_{n}=\xi^{n} \frac{d}{d \xi} \frac{1}{\xi^{n}}=\frac{d}{d \xi}-n \xi^{-1}, \quad D_{n}^{\#}=-\frac{1}{\xi^{n}} \frac{d}{d \xi} \xi^{n}=-\frac{d}{d \xi}-n \xi^{-1}, \quad n=1, \ldots
$$


are generated by zero energy non-physical eigenstates $\xi^{n}$ of $H_{n-1}^{\alpha}, H_{n-1}^{\alpha} \xi^{n}=0$. The bounded but not square-integrable physical eigenstates of $H_{n}^{\alpha}$ from the continuous part of the spectrum are obtained by applying $D_{n}$ to the corresponding eigenstates of $H_{n-1}^{\alpha}$ of the same nature, $\psi_{n, \pm k}^{\alpha}=D_{n} \psi_{n-1, \pm k}^{\alpha}, k>0$. These states can be constructed iteratively from the plane wave eigenstates of the free particle model,

$$
\psi_{n, \pm k}^{\alpha}(x)=\hat{\mathcal{D}}_{n} e^{ \pm i k \xi}
$$

Here we define differential operators of order $n$ :

$$
\hat{\mathcal{D}}_{n}=D_{n} D_{n-1} \ldots D_{1}, \quad \hat{\mathcal{D}}_{n}^{\#}=D_{1}^{\#} \ldots D_{n-1}^{\#} D_{n}^{\#} .
$$

The square-integrable zero energy ground state $\psi_{n, 0}^{\alpha}=\xi^{-n}$ of $H_{n}^{\alpha}$ is obtained from a singlet zero energy ground state $\psi_{0,0}=1$ of $H_{0}: \psi_{n, 0}^{\alpha} \propto \hat{\mathcal{D}}_{n} 1$.

The operators (2.21) intertwine $H_{n}^{\alpha}$ directly with $H_{0}$,

$$
\hat{\mathcal{D}}_{n} H_{0}=H_{n}^{\alpha} \hat{\mathcal{D}}_{n}, \quad \hat{\mathcal{D}}_{n}^{\#} H_{n}^{\alpha}=H_{0} \hat{\mathcal{D}}_{n}^{\#},
$$

and correspond to the Darboux-Crum transformation of order $n$ of the free particle based on non-physical eigenstate $\xi$ of $H_{0}$ and its certain $n-1$ Jordan states, see below. They allow us to construct a nontrivial integral of the system $H_{n}^{\alpha}$ in the form of the dressed momentum operator of the free particle,

$$
\mathcal{P}_{n}^{\alpha}=-i \hat{\mathcal{D}}_{n} \frac{d}{d x} \hat{\mathcal{D}}_{n}^{\#}, \quad\left[\mathcal{P}_{n}^{\alpha}, H_{n}^{\alpha}\right]=0 .
$$

Being differential operator of order $2 n+1$, it distinguishes the left- and right-moving eigenstates (2.20) of $H_{n}^{\alpha}$,

$$
\mathcal{P}_{n}^{\alpha} \psi_{n, \pm k}^{\alpha}= \pm k^{2 n+1} \psi_{n, \pm k}^{\alpha},
$$

and satisfies the Burchnall-Chaundy, supersymmetry-type relation

$$
\left(\mathcal{P}_{n}^{\alpha}\right)^{2}=\left(H_{n}^{\alpha}\right)^{2 n+1}
$$

Its kernel of dimension $2 n+1$ is

$$
\operatorname{ker} \mathcal{P}_{n}^{\alpha}=\operatorname{span}\left\{\xi^{-n}, \xi^{-n+2}, \ldots, \xi^{3 n-2}, \xi^{3 n}\right\} .
$$

Except the zero energy ground state $\xi^{-n}$, all other states from the kernel are not eigenstates of $H_{n}^{\alpha}$ but satisfy the relations

$$
H_{n}^{\alpha} \xi^{-n+2 l}=2 l(2 n-2 l+1) \xi^{-n+2 l-2}, \quad\left(H_{n}^{\alpha}\right)^{l+1} \xi^{-n+2 l}=0, \quad l=0, \ldots, 2 n .
$$

Each of the states $\xi^{-n+2 l}$ with $l=1, \ldots, 2 n$ is the Jordan state of $H_{n}^{\alpha}$ of the corresponding order $l+1$.

The partner (2.1) of zero energy ground state $\psi_{n, 0}^{\alpha}=\xi^{-n}$ of $H_{n}^{\alpha}$ is a non-physical state $\xi^{n+1}$. It does not belong to the kernel (2.26), but the action of $\mathcal{P}_{n}^{\alpha}$ transforms it into the 
physical ground state $\xi^{-n}$. It can be obtained from a linear combination of the physical eigenstates from the continuous part of the spectrum of $H_{n}^{\alpha}$ :

$$
\lim _{k \rightarrow 0} \frac{1}{2 i k^{2 n+1}}\left(\psi_{n, k}^{\alpha}-\psi_{n,-k}^{\alpha}\right)=C_{n} \xi^{n+1},
$$

where $C_{n}$ is a constant coefficient. It also can be obtained from the plane wave solutions of the free particle by employing relation (2.20).

In accordance with relations $(2.22)$ and $(2.21)$, the potential $V_{n}(x)=\frac{n(n+1)}{\xi^{2}}$ can be presented in the form

$$
V_{n}(x)=-2 \frac{d^{2}}{d x^{2}}\left(\ln \mathbb{W}_{n}(\xi)\right),
$$

where $\mathbb{W}_{n}(\xi):=W\left(\xi, \xi^{3}, \ldots, \xi^{2 n+1}\right)$ is the Wronskian of the states $\xi, \xi^{3}, \ldots, \xi^{2 n+1}$. Here the states $\xi^{2 r+1}$ with $r=1, \ldots, n-1$ are the Jordan states of $H_{0}$ of zero energy of the corresponding order: $H_{0} \xi^{2 r+1}=-2 r(2 r+1) \xi^{2 r-1},\left(H_{0}\right)^{r+1} \xi^{2 r+1}=0$. Such a transformation based on Jordan states corresponds to a confluent case of some Darboux-Crum transformation [35, 43, 44]. For the eigenstates $\psi_{n, \pm k}^{\alpha}$ of $H_{n}^{\alpha}$ from $(2.20)$ we have (up to a multiplicative constant factor) an alternative representation :

$$
\psi_{n, \pm k}^{\alpha}=\frac{W\left(\xi, \xi^{3}, \ldots, \xi^{2 n+1}, e^{ \pm i k \xi}\right)}{\mathbb{W}_{n}(\xi)} .
$$

The case $k=0$ is included in (2.30), and corresponds to generation of the unique squareintegrable zero energy eingenstate $\psi_{n, 0}^{\alpha}=\xi^{-n}$ of $H_{n}^{\alpha}$ from the eigenstate $\psi_{0,0}=1$ of $H_{0}$. Note that the arguments $\xi^{2 j+1}$ in the Wronskians also can be presented in the form $\xi^{2 j+1}=$ $\left.(-i)^{2 j+1} \frac{\partial^{2 j+1}}{\partial k^{2 j+1}} e^{i k \xi}\right|_{k=0}$ in terms of the plane wave eigenstates of the free particle. This corresponds to the generalized Wronskian formula for solutions of the KdV equation considered in ref. [26] that, in turn, can be obtained via confluent Darboux-Crum transformations [35].

The nature of the family of the $\mathcal{P} \mathcal{T}$-symmetric systems (2.16) is rather peculiar. Like any finite-gap system, each of the systems (2.16) possesses the corresponding Lax-Novikov integral of motion (2.23) that is a differential operator of order $2 n+1$. Each of these systems is reflectionless : the plane wave states $e^{i k x}$ and $e^{-i k x}$ of the free particle $H_{0}$ are transformed into the deformed plane wave eigenstates (2.20) of $H_{n}^{\alpha}$ which propagate to the left or to the right, and are distinguished by the Lax-Novikov integral, see eq. (2.24). The two indicated properties are also characteristic for any system with a multi-soliton potential as for their simplest one-soliton representative (2.5). Unlike the indicated conventional reflectionless systems, the $\mathcal{P} \mathcal{T}$-symmetric systems (2.16) are perfectly invisible. Namely, as follows from (2.21) and (2.19), the eigenstates (2.20) corresponding to the upper sign case in the asymptotic region $x \rightarrow-\infty$ have the form of the plane waves $C e^{i k x}$. In the asymptotic region $x \rightarrow+\infty$ these states have exactly the same form of the plane waves $e^{i k x}$ multiplied by the same constant factor $C$. A similar picture is valid for solutions that correspond to the lower sign in (2.20) and have the asymptotic form $C e^{-i k x}$ in both regions $x \rightarrow+\infty$ and $x \rightarrow-\infty$. The phase shift produced by a nontrivial potential in (2.16) is therefore equal to zero $(\bmod 2 \pi)$. The transmission amplitude is equal to one, and any of these systems is indeed perfectly invisible. 
The potential $V_{n}(x)=\frac{n(n+1)}{\xi^{2}}$ of $H_{n}^{\alpha}$ is a stationary $\mathcal{P} \mathcal{T}$-symmetric solution of the corresponding higher order equation of the KdV hierarchy. For instance, the potential $V_{2}(x)=\frac{6}{\xi^{2}}$ is a stationary solution to the equation

$$
u_{t}+30 u^{2} u_{x}-20 u_{x} u_{x x}-10 u u_{x x x}+u_{x x x x x}=0 .
$$

\section{$2.2 \mathcal{P} \mathcal{T}$-symmetric rational extensions of the $\mathcal{P} \mathcal{T}$-regularized Calogero sys- tems}

The shape-invariant family (2.16) is not a unique set of perfectly invisible $\mathcal{P} \mathcal{T}$-symmetric systems that can be constructed by applying Darboux transformations to the free particle. A simple example of such a system of the form different from (2.16) can be obtained by taking a wave function

$$
\psi_{\alpha, \gamma}^{(1)}=\gamma \xi^{-1}+\xi^{2}
$$

as a seed state for the Darboux transformation. This is a linear combination of the bound state $\xi^{-1}$ of zero energy of the system $H_{1}^{\alpha}$ and of its non-physical partner in the sense of (2.1), where $\gamma$ is a constant. Requiring that $\gamma$ is purely imaginary, the seed state (2.32) will be $\mathcal{P} \mathcal{T}$-invariant. Via a $\mathcal{P} \mathcal{T}$-odd superpotential

$$
\mathcal{W}_{\alpha, \gamma}^{(1)}=-\frac{d}{d x}\left(\ln \psi_{\alpha, \gamma}^{(1)}\right)=\frac{1}{\xi}-\frac{3 \xi^{2}}{\xi^{3}+\gamma},
$$

one can generate two super-partner potentials $V_{ \pm}=\left(\mathcal{W}_{\alpha, \gamma}^{(1)}\right)^{2} \pm\left(\mathcal{W}_{\alpha, \gamma}^{(1)}\right)^{\prime}$, where $V_{-}=2 \xi^{-2}$ and

$$
V_{+}^{(1)}(x ; \alpha, \gamma)=6 \xi \frac{\xi^{3}-2 \gamma}{\left(\xi^{3}+\gamma\right)^{2}}=\frac{6}{\xi^{2}}-6 \gamma \frac{4 \xi^{3}+\gamma}{\xi^{2}\left(\xi^{3}+\gamma\right)^{2}} .
$$

Potential (2.33) is a stationary $\mathcal{P} \mathcal{T}$-symmetric solution to the equation (2.31), which is non-singular on $\mathbb{R}^{1}$ function provided

$$
\gamma=i \nu \alpha^{3}, \quad \nu \in \mathbb{R}^{1}, \quad \nu \neq-8,1 .
$$

On the other hand, if we put in (2.33)

$$
\gamma=\gamma(t)=12 t+i \nu \alpha^{3}, \quad \nu \in(1, \infty)
$$

we obtain a complex $\mathcal{P} \mathcal{T}$-symmetric solution $V_{+}^{(1)}(x, t ; \alpha)$ of the $\mathrm{KdV}$ equation (2.4), which is non-singular for $t \in(-\infty, \infty)$. If in this time-dependent solution we put $\alpha=0$, it takes the form of the well known rational singular solution $u(x, t)=6 x \frac{x^{3}-24 t}{\left(x^{3}+12 t\right)^{2}}$ to the $\mathrm{KdV}$ equation (2.4) [45]. The permitted values of the parameter $\gamma$ indicated in (2.34) and (2.35) guarantee that the amplitude of the wave function (2.32) nowhere turns into zero. Note that the fact that the potential $V_{+}^{(1)}(x, t ; \alpha)$ satisfies simultaneously the timedependent $\mathrm{KdV}$ equation (2.4) and the higher order ordinary differential equation $30 u^{2} u_{x}-$ $20 u_{x} u_{x x}-10 u u_{x x x}+u_{x x x x x}=0$ being a stationary case of (2.31) corresponds to a general property mentioned in section 1 in relation to the Novikov equation.

The variation of the form of real and imaginary parts of the potential $(2.33),(2.34)$ as a stationary $\mathcal{P} \mathcal{T}$-symmetric solution $(t=0)$ to the higher order $\mathrm{KdV}$ equation (2.31) are illustrated by figures 1 and 2 for various values of the parameters $\alpha$ and $\nu$. 

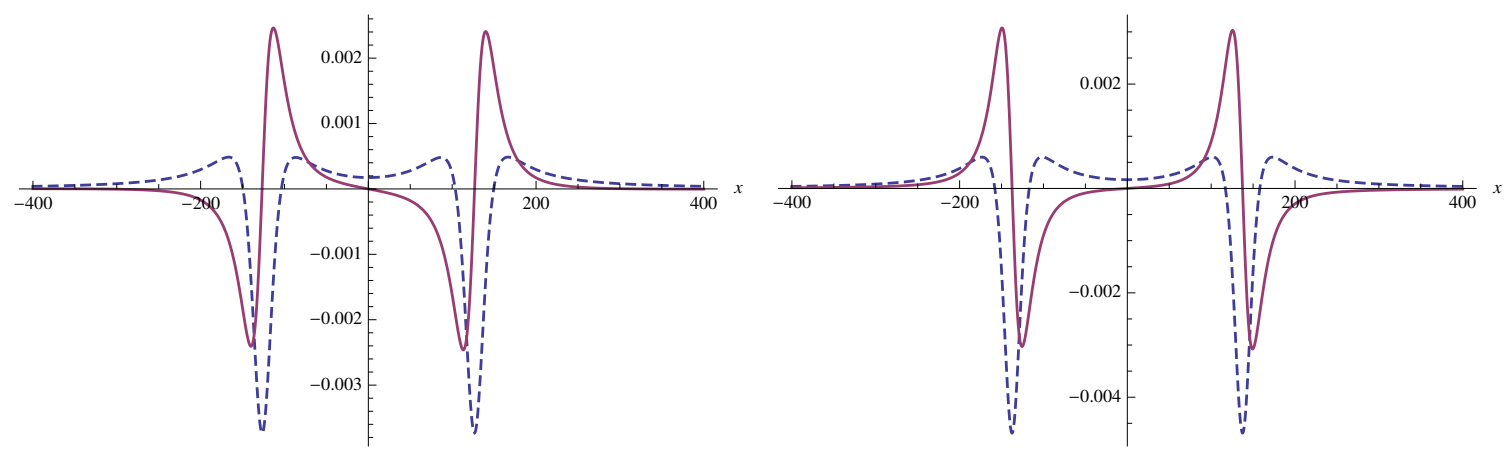

Figure 1. Real (shown by dashed lines) and imaginary (shown by continuous lines) parts of potential (2.33) as a complex $\mathcal{P} \mathcal{T}$-symmetric stationary $(t=0)$ solution to eq. (2.31) at $\alpha=50$ and $\nu=-25$ (on the left) and $\alpha=100, \nu=-4$ (on the right). The graph of imaginary part undergoes a flip as the parameter $\nu$ passes through a critical value -8 .
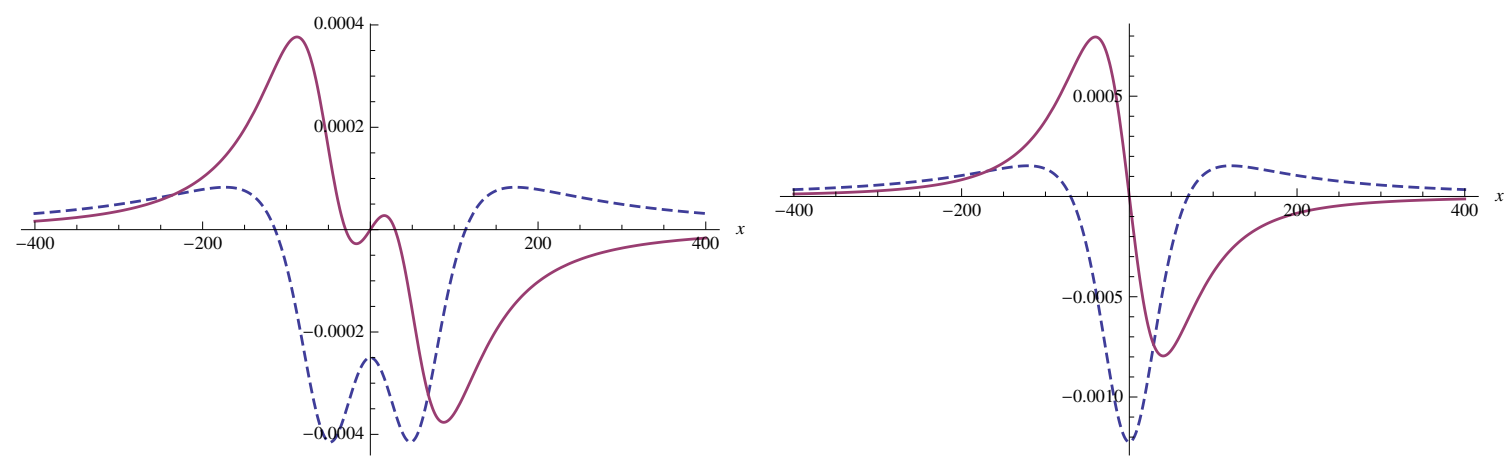

Figure 2. Real and imaginary parts of (2.33) of a stationary case (2.34) at $\alpha=100$, and $\nu=-0.2$ (on the left) and $\nu=0$ (on the right).

The evolution of this potential in the time-dependent case (2.35) as a $\mathcal{P} \mathcal{T}$-symmetric solution to the $\mathrm{KdV}$ equation (2.4) is shown on figure 3. It is interesting to note that near the critical value $\nu=1$ of the parameter $\nu$, the graph of real part of the potential demonstrates a singular $\delta$-function type behaviour while the imaginary part undergoes a flip and has a typical $\delta^{\prime}$ form. This critical value is at the very lower edge of the infinite interval (2.35) of the permitted values for the parameter $\nu$ in the time-dependent case. As a consequence, for the values of the parameter $\nu$ close to the critical value $\nu=1$, the inverted potential $-V_{+}^{(1)}(x ; \alpha, \gamma(t))=u(x, t)$ reveals a behaviour typical for rogue (extreme) waves that is seen already from figure 3 .

On the base of the function (2.32), one can construct the first order differential operators

$$
D_{\alpha, \gamma}^{(1)}=\psi_{\alpha, \gamma}^{(1)} \frac{d}{d x} \frac{1}{\psi_{\alpha, \gamma}^{(1)}}=\frac{d}{d x}+\mathcal{W}_{\alpha, \gamma}^{(1)}, \quad D_{\alpha, \gamma}^{(1) \#}=\frac{1}{\psi_{\alpha, \gamma}^{(1)}} \frac{d}{d x} \psi_{\alpha, \gamma}^{(1)}=-\frac{d}{d x}+\mathcal{W}_{\alpha, \gamma}^{(1)},
$$

which factorize the pair of Hamiltonians (2.9) and $H_{\alpha, \gamma}^{(1)}=-\frac{d^{2}}{d x^{2}}+V_{+}^{(1)}(x ; \alpha, \gamma)$ : $D_{\alpha, \gamma}^{(1) \#} D_{\alpha, \gamma}^{(1)}=H_{1}^{\alpha}, D_{\alpha, \gamma}^{(1)} D_{\alpha, \gamma}^{(1) \#}=H_{\alpha, \gamma}^{(1)}$, and intertwine them: $D_{\alpha, \gamma}^{(1)} H_{1}^{\alpha}=H_{\alpha, \gamma}^{(1)} D_{\alpha, \gamma}^{(1)}$, 

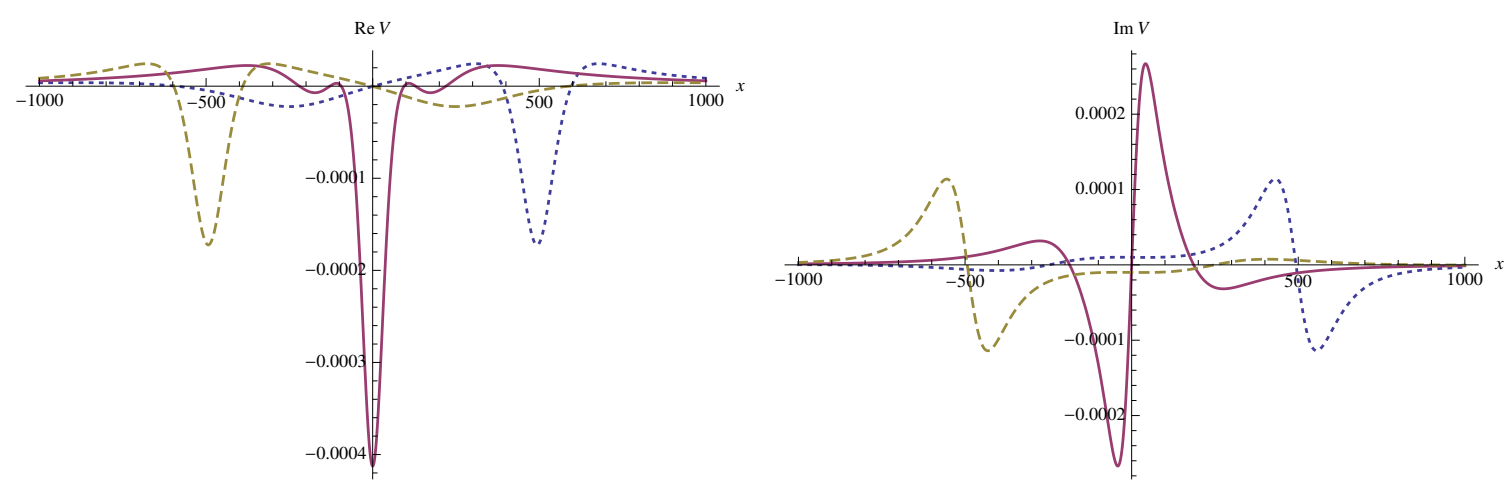

Figure 3. Evolution of real (on the left) and imaginary (on the right) parts of potential (2.33), (2.35) as a complex $\mathcal{P} \mathcal{T}$-symmetric solution of the $\mathrm{KdV}$ equation (2.4) at $\alpha=100, \nu=5$; dashed lines: $t=-10^{7}$, continuous lines: $t=0$, dotted lines: $t=10^{7}$.

$D_{\alpha, \gamma}^{(1) \#} H_{\alpha, \gamma}^{(1)}=H_{1}^{\alpha} D_{\alpha, \gamma}^{(1) \#}$. In correspondence with these relations, the bounded eigenstates of the system $H_{\alpha, \gamma}^{(1)}$ are constructed from the plane wave eigenstates of the free particle system, $\psi_{\alpha, \gamma, \pm k}^{(1)}=D_{\alpha, \gamma}^{(1)} D_{1} e^{ \pm i k \xi}$, where the operator $D_{1}$ is given by eq. (2.8). The unique quadratically integrable eigenstate $\psi_{\alpha, \gamma, 0}^{(1)}(x)=\frac{\xi}{\xi^{3}+\gamma}$ of zero eigenvalue of the system $H_{\alpha, \gamma}^{(1)}$ corresponds to the limit case $k=0$ of the bounded eigenstates $\psi_{\alpha, \gamma, \pm k}^{(1)}$. It is generated by applying the second order operator $D_{\alpha, \gamma}^{(1)} D_{1}$ to the bounded eigenstate $\psi_{0,0}=1$ of the free particle $H_{0}$, or, equivalently, by applying the first order operator $D_{\alpha, \gamma}^{(1)}$ to the ground state $\xi^{-1}$ of zero energy of $H_{1}^{\alpha}$. This state is annihilated by the Lax-Novikov integral

$$
\mathcal{P}_{\alpha, \gamma}^{(1)}=-i D_{\alpha, \gamma}^{(1)} D_{1} \frac{d}{d x} D_{1}^{\#} D_{\alpha, \gamma}^{(1) \#}
$$

$\left[H_{\alpha, \gamma}^{(1)}, \mathcal{P}_{\alpha, \gamma}^{(1)}\right]=0$, that satisfies the Burchnall-Chaundy relation $\left(\mathcal{P}_{\alpha, \gamma}^{(1)}\right)^{2}=\left(H_{\alpha, \gamma}^{(1)}\right)^{5}$, and distinguishes the bounded eigenstates $\psi_{ \pm k}^{\alpha, \gamma}: \mathcal{P}_{\alpha, \gamma}^{(1)} \psi_{ \pm k}^{\alpha, \gamma}= \pm k^{5} \psi_{ \pm k}^{\alpha, \gamma}$. The potential (2.33) can be produced directly from the free particle system via the relation

$$
V_{+}^{(1)}(x ; \alpha, \gamma)=-2 \frac{d^{2}}{d x^{2}}\left(\ln W\left(\xi,-\gamma+\xi^{3}\right)\right),
$$

that corresponds to the second order Darboux-Crum transformation. Here the first argument of the Wronskian is the non-physical eigenstate $\xi$ of zero eigenvalue of $H_{0}$, whereas the second argument corresponds to a linear combination of the eigenstate $\psi_{0,0}=1$ and the Jordan state $\xi^{3}$ of the second order, $\left(H_{0}\right)^{2} \xi^{3}=0$.

As yet another example of the $\mathcal{P} \mathcal{T}$-symmetric perfectly invisible system we present here the system described by the potential

$$
V_{+}^{(2)}(x ; \alpha, \gamma)=\frac{2}{\xi^{2}}+10 \xi^{3} \frac{\xi^{5}-4 \gamma}{\left(\xi^{5}+\gamma\right)^{2}}=\frac{12}{\xi^{2}}-10 \gamma \frac{6 \xi^{5}+\gamma}{\xi^{2}\left(\xi^{5}+\gamma\right)^{2}},
$$

where $\gamma$ is, again, a purely imaginary parameter. This potential can be produced from the system $H_{2}^{\alpha}$ by constructing the Darboux transformation based on the state

$$
\psi_{\alpha, \gamma}^{(2)}=\gamma \xi^{-2}+\xi^{3},
$$



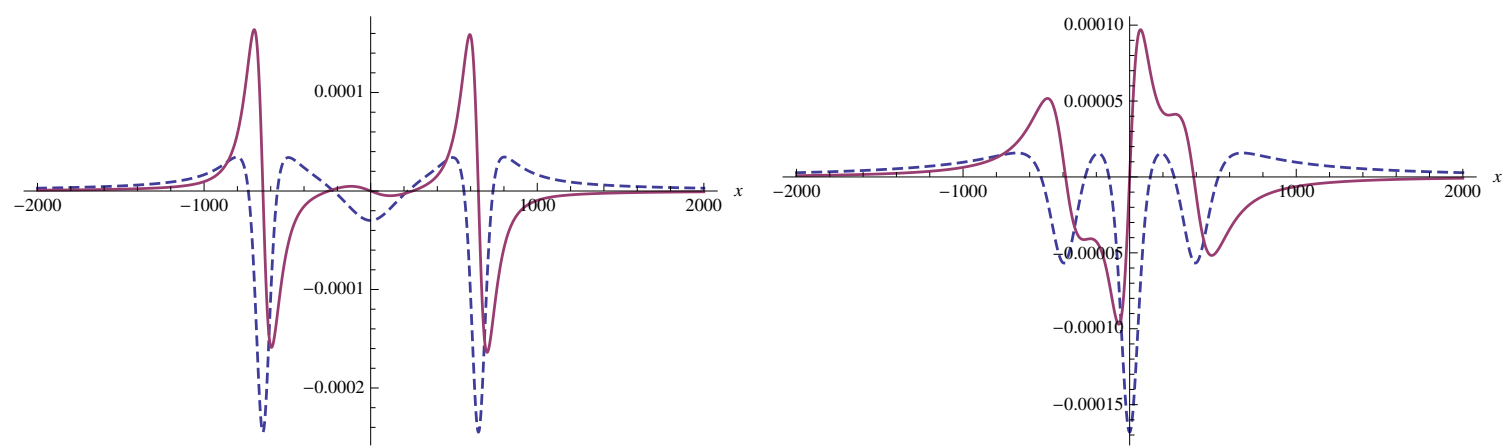

Figure 4. Real (shown by dashed lines) and imaginary (shown by continuous lines) parts of potential (2.39), (2.41) as a complex $\mathcal{P} \mathcal{T}$-symmetric stationary solution to eq. (2.31) at $\alpha=300$ and $\nu=-60$ (on the left) and $\nu=-5$ (on the right).

that is a linear combination of the quadratically integrable zero energy eigenstate $\xi^{-2}$ of $H_{2}^{\alpha}$ and of its non-physical partner $\xi^{3}$. Equivalently, the perfectly transparent potential (2.39) can be produced by the relation of the form (2.38) with the Wronskian changed for $W\left(\xi, \xi^{3}, \frac{8}{3} \gamma+\xi^{5}\right)$, where $\xi^{5}$ in the last argument corresponds to the Jordan state of $H_{0}$ of the third order: $\left(H_{0}\right)^{3} \xi^{5}=0$. The potential (2.39) is a stationary $\mathcal{P} \mathcal{T}$-symmetric solution to the equation (2.31), which is non-singular provided the following restriction on the values of the parameter $\gamma$ is introduced:

$$
\gamma=i \nu \alpha^{5}, \quad \nu \in \mathbb{R}^{1}, \quad \nu \neq-1,4,
$$

under which the wave function (2.40) nowhere turns into zero on real line $x \in \mathbb{R}^{1}$.

The substitution

$$
\gamma=\gamma(t)=-720 t+i \nu \alpha^{5}, \quad \nu \in(24, \infty)
$$

in (2.39) transforms the $\mathcal{P} \mathcal{T}$-symmetric stationary solution $V_{+}^{(2)}(x ; \alpha, \gamma)$ of $(2.31)$ into the $\mathcal{P} \mathcal{T}$-symmetric function $V_{+}^{(2)}(x, t ; \alpha)$ that is a time-dependent $\mathcal{P} \mathcal{T}$-symmetric solution of the same equation (2.31) to be non-singular for all values of $t \in(-\infty, \infty)$. In the case $\gamma=0$, potential (2.39) reduces to $\frac{12}{\xi^{2}}$, that is a potential of the $\mathcal{P} \mathcal{T}$-symmetric perfectly transparent system (2.16) with $n=3$.

The time dependence (2.35) and (2.42) for the corresponding potentials considered here as well as in a general case can be fixed by exploiting the covariance under Darboux transfromations of the Lax representation for the KdV equation and for higher equations of the hierarchy mentioned in section 1 .

The form of potential (2.39) for the stationary and time-dependent cases is illustrated by figures 4,5 and 6 . Note that unlike the case corresponding to the potential (2.33), the critical value $\nu=4$ here cannot be approached by the time-dependent solutions (2.42).

The corresponding Hamiltonian operator $H_{\alpha, \gamma}^{(2)}=-\frac{d^{2}}{d x^{2}}+V_{+}^{(2)}(x ; \alpha, \gamma)$ is factorized, $H_{\alpha, \gamma}^{(2)}=D_{\alpha, \gamma}^{(2)} D_{\alpha, \gamma}^{(2) \#}$, and we also have $D_{\alpha, \gamma}^{(2) \#} D_{\alpha, \gamma}^{(2)}=H_{2}^{\alpha}$. Here the factorizing operators have the form similar to that in (2.36) with the generating function changed for $\psi_{\alpha, \gamma}^{(2)}$, and with 

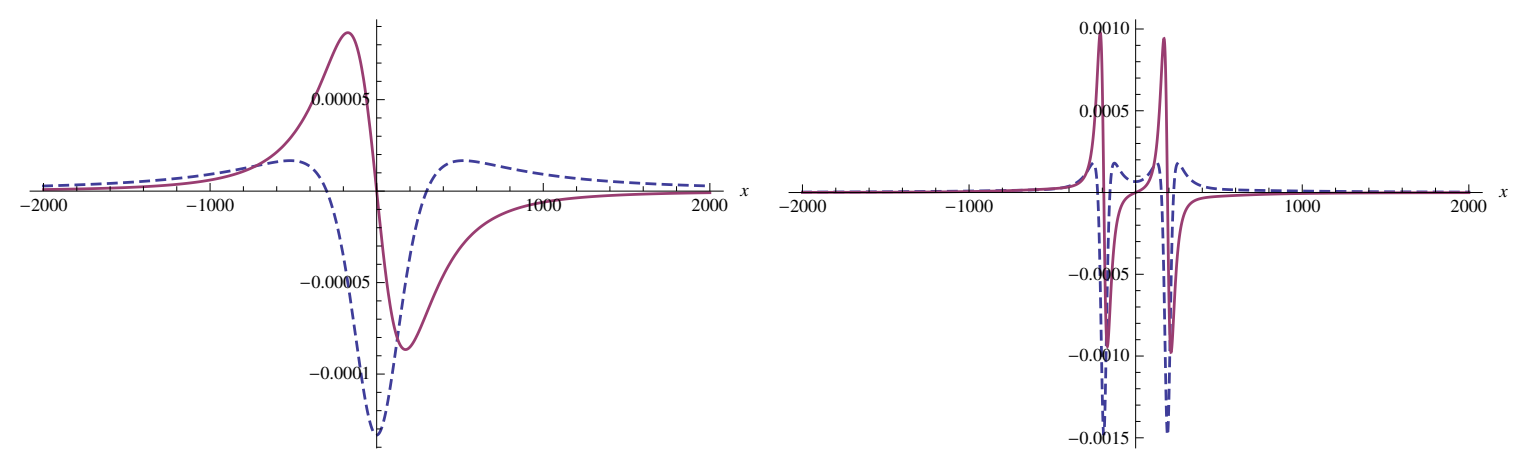

Figure 5. Real and imaginary parts of potential (2.39), (2.41) as a complex $\mathcal{P} \mathcal{T}$-symmetric stationary $(t=0)$ solution to eq. (2.31) at $\alpha=300$ and $\nu=0$ (on the left) and $\nu=1.5$ (on the right).
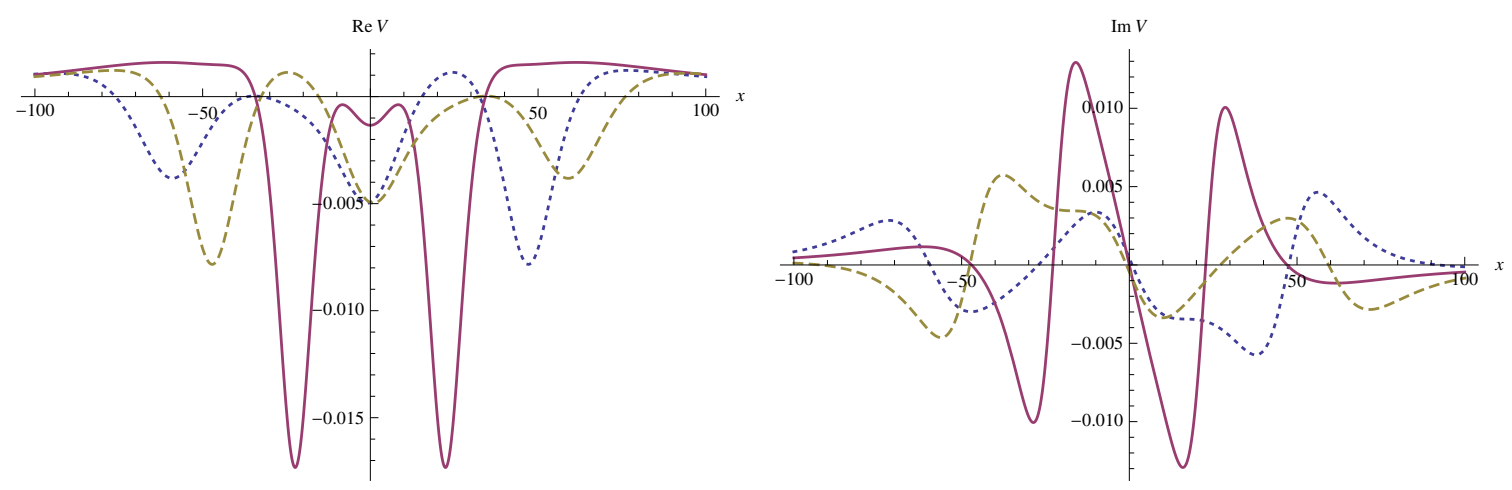

Figure 6. Evolution of real (on the left) and imaginary (on the right) parts of potential (2.39), (2.42) as a complex $\mathcal{P} \mathcal{T}$-symmetric solution to eq. (2.39) at $\alpha=20, \nu=25$; dashed lines: $t=-10^{6}$, continuous lines: $t=0$, dotted lines: $t=10^{6}$.

the superpotential replaced by

$$
\mathcal{W}_{\alpha, \gamma}^{(2)}=\frac{2}{\xi}-\frac{5 \xi^{4}}{\gamma+\xi^{5}}
$$

The perfectly transparent $\mathcal{P} \mathcal{T}$-symmetric system $H_{\alpha, \gamma}^{(2)}$ is characterized by the Lax-Novikov integral

$$
\mathcal{P}_{\alpha, \gamma}^{(2)}=-i D_{\alpha, \gamma}^{(2)} D_{2} D_{1} \frac{d}{d x} D_{1}^{\#} D_{2}^{\#} D_{\alpha, \gamma}^{(2) \#}
$$

This differential operator of order seven satisfies the relations $\left[H_{\alpha, \gamma}^{(2)}, \mathcal{P}_{\alpha, \gamma}^{(2)}\right]=0$ and $\left(\mathcal{P}_{\alpha, \gamma}^{(2)}\right)^{2}=$ $\left(H_{\alpha, \gamma}^{(2)}\right)^{7}$. The bounded eigenstates of the system $H_{\alpha, \gamma}^{(2)}$ are constructed from the plane wave eigenstates of the free particle system by applying to them the third order differentia operator $D_{\alpha, \gamma}^{(2)} D_{2} D_{1}$ that intertwines $H_{0}$ with $H_{\alpha, \gamma}^{(2)}$. The unique quadratically integrable eigenstate $\psi_{\alpha, \gamma, 0}^{(2)}=\frac{\xi^{2}}{\xi^{5}+\gamma}$ of the system $H_{\alpha, \gamma}^{(2)}$ of zero eigenvalue is given by application of the same third order differential operator to the eigenstate $\psi_{0,0}=1$ of $H_{0}$. Equivalently, $\psi_{\alpha, \gamma, 0}^{(2)}$ is produced by applying the first order differential operator $D_{\alpha, \gamma}^{(2)}$ to the ground state $\xi^{-2}$ of zero energy of the system $H_{2}^{\alpha}$. It is annihilated by the Lax-Novikov integral (2.43). 
Potential (2.33) at $\gamma=0$ is reduced to the $\mathcal{P} \mathcal{T}$-symmetric potential $6 \xi^{-2}$, while for $|\gamma| \rightarrow \infty$ it turns into zero. Potential (2.39) at $\gamma=0$ takes a form $12 \xi^{-2}$, and in the limit $|\gamma| \rightarrow \infty$ it is reduced to $2 \xi^{-2}$. Therefore, the quantum system with potential (2.33) interpolates between the free particle and the $\mathcal{P} \mathcal{T}$-symmetric system (2.16) with $n=2$, while the system with potential (2.39) interpolates between the perfectly transparent $\mathcal{P} \mathcal{T}$ symmetric systems (2.16) with $n=1$ and $n=3$. Let us stress, however, that continuous variation of $\gamma$ between 0 and $\infty$ is impossible since, according to (2.34) and (2.41), we have to pass through the excluded purely imaginary values of $\gamma$ where the corresponding potentials loose their non-singular in $x \in \mathbb{R}^{1}$ nature.

It is interesting to note here what happens with the zero energy ground states of the systems $H_{\alpha, \gamma}^{(1)}$ and $H_{\alpha, \gamma}^{(2)}$ in the indicated limit cases. The ground state $\psi_{\alpha, \gamma, 0}^{(1)}=\frac{\xi}{\xi^{3}+\gamma}$ of $H_{\alpha, \gamma}^{(1)}$ in the case $\gamma=0$ takes the form of the ground state $\xi^{-2}$ of $H_{2}^{\alpha}$, whereas the ground state $\psi_{\alpha, \gamma, 0}^{(2)}=\frac{\xi^{2}}{\xi^{5}+\gamma}$ of $H_{\alpha, \gamma}^{(2)}$ at $\gamma=0$ reduces to the ground state $\xi^{-3}$ of $H_{3}^{\alpha}$. In the limit $|\gamma| \rightarrow \infty$, however, both states turn into zero. These states mutliplied by $\gamma$ in the indicated limit are transformed into the states $\xi$ and $\xi^{2}$, which are the zero energy non-physical eigenstates of $H_{0}$ and $H_{1}^{\alpha}$, respectively. On the other hand, the non-physical partner $\widetilde{\psi_{\alpha, \gamma, 0}^{(1)}}$ of the ground state $H_{\alpha, \gamma}^{(1)}$ given by $(2.1)$ and rescaled by inclusion of the multiplication factor $\gamma^{-1}$ transforms in the limit $|\gamma| \rightarrow \infty$ into the ground state $\psi_{0,0}=1$ of the system $H_{0}$. In the same way, the non-physical eigenstate $\gamma^{-1} \widetilde{\psi_{\alpha, \gamma, 0}^{(2)}}$ of $H_{\alpha, \gamma}^{(2)}$ of zero eigenvalue in the limit $|\gamma| \rightarrow \infty$ is transformed into the ground state $\xi^{-1}$ of the system $H_{1}^{\alpha}$. Therefore, in the limit $|\gamma| \rightarrow \infty$, we have a kind of transmutation of physical and non-physical eigenstates of zero energy of the systems $H_{\alpha, \gamma}^{(1)}$ and $H_{\alpha, \gamma}^{(2)}$ into, respectively, non-physical and physical states of zero eigenvalue of the corresponding limit systems $H_{0}$ and $H_{1}^{\alpha}$.

In conclusion of this section we note that the quantum systems given by the $\mathcal{P} \mathcal{T}$ symmetric potentials (2.33) and (2.39), unlike the $\mathcal{P} \mathcal{T}$-regularized Calogero systems (2.16), are not conformal invariant. This can be related to the difference in the properties of the wave functions (2.32) and (2.40) in comparison with those of wave functions of the form $\xi^{2 n+1}$ with respect to the scale, $\xi \rightarrow \lambda \xi$, and inversion, $\xi \rightarrow 1 / \xi$, transformations.

\section{3 (1+1)-dimensional conformal field theoretical kinks seen from confor- mal quantum mechanics}

In this section we discuss how the Hamiltonians $H_{1}^{\alpha}$ and $H_{2}^{\alpha}$ governing the dynamics in conformal invariant $\mathcal{P} \mathcal{T}$-symmetric quantum mechanical systems also determine the fluctuation spectra around the singular kinks arising as traveling waves in the Liouville and $\mathrm{SU}(3)$ conformal Toda systems in field theory. This is remarkable because the conformal group which is infinite dimensional in the two-dimensional Minkowskian setting contracts to a finite subgroup in conformal quantum mechanics, see also [46, 47]. 


\subsection{Kinks in Liouville field theory}

The classical dynamics of Liouville field theory $[48,49]$ is governed by the action:

$$
S[\phi]=\int d t \int d x\left\{\frac{1}{2}\left(\frac{\partial \phi}{\partial t}\right)^{2}-\frac{1}{2}\left(\frac{\partial \phi}{\partial x}\right)^{2}-\frac{m^{2}}{\beta^{2}} \cdot \exp [\beta \phi(t, x)]\right\},
$$

where $m^{2}$ is a parameter of dimension (length) $)^{-2}$ in the natural system of units $\hbar=c=1$. The coupling constant $\beta>0$, however, is dimensionless. The Liouville field equation

$$
\frac{\partial^{2} \phi}{\partial t^{2}}(t, x)-\frac{\partial^{2} \phi}{\partial x^{2}}(t, x)+\frac{m^{2}}{\beta} \cdot \exp [\beta \phi(t, x)]=0
$$

is completely integrable. The general solution of (3.2) is obtained, in terms of arbitrary functions of the light-cone coordinates $x_{ \pm}=\frac{1}{\sqrt{2}}(t \pm x)$, via Bäcklund transformations starting from the general solution of the free wave equation. In these variables the wave and Liouville equations read respectively:

$$
2 \partial_{+} \partial_{-} \phi_{0}\left(x_{+}, x_{-}\right)=0, \quad 2 \partial_{+} \partial_{-} \phi\left(x_{+}, x_{-}\right)+\frac{m^{2}}{\beta} \exp \left[\beta \phi\left(x_{+}, x_{-}\right)\right]=0,
$$

where we have denoted $\partial_{ \pm}=\frac{\partial}{\partial x_{ \pm}}$. Equations (3.3) are thus invariant under the coordinate transformations $x_{+}^{\prime}=f_{+}\left(x_{+}\right), x_{-}^{\prime}=f_{-}\left(x_{-}\right)$, with $f_{ \pm}\left(x_{ \pm}\right)$arbitrary functions of their arguments, accomponied by the field redefinition $\phi^{\prime}\left(x_{+}^{\prime}, x_{-}^{\prime}\right)=-\frac{1}{\beta} \ln \left[\partial_{+} f_{+}\left(x_{+}\right) \partial_{-} f_{-}\left(x_{-}\right)\right]+$ $\phi\left(x_{+}, x_{-}\right)$[49]. In sum, the Liouville equation is invariant under the infinite-dimensional conformal group, whose Lie algebra is the Virasoro algebra.

There is a static homogeneous solution of equation $(3.3): \phi^{0}=-\infty$. Linearization of the Liouville equation around the constant solution, $\phi(t, x)=\phi^{0}+\delta \phi(t, x)$,

$$
\left(\frac{\partial^{2}}{\partial t^{2}}-\frac{\partial^{2}}{\partial x^{2}}\right) \delta \phi(t, x)=\mathcal{O}\left(\delta^{2}\right) \Rightarrow \delta \phi_{\omega}(t, x)=e^{i \omega t} e^{i k x}, \quad \omega^{2}=k^{2},
$$

shows a semi-classical picture of the Liouville quanta as massless bosons. Liouville quantum theory in full exhibits a very particular structure as a quantum conformal system, see e.g. [48]. Our goal here, however, is the search for traveling wave type of solutions, that is, we make the ansatz $\phi(t, x)=f\left(\lambda \cdot \frac{x-v t-x_{0}}{\sqrt{1-v^{2}}}\right), \lambda \in \mathbb{R}^{+},|v|^{2}<1, x_{0} \in \mathbb{R}$, which can be obtained from $\phi(t, x)=f(x)$ by applying to $x$, first, a scale transformation, $x^{\prime}=\lambda x$, and then, a Poincaré transformation parametrized by $\left(v, x_{0}\right)$. Thus, in the frame $\left(\lambda=1, v=0, x_{0}=0\right)$, one has to solve the first-order ordinary differential equation

$$
\frac{d f}{d x}=\sqrt{2 \frac{m^{2}}{\beta^{2}} \cdot \exp [\beta f(x)]} .
$$

The invariance under dilatations, Lorentz transformations and spatial translations provides the family of singular kinks

$$
f_{K}(t, x)=\frac{1}{\beta} \log \left[\frac{2}{m^{2} \lambda^{2}\left(\frac{x-v t-x_{0}}{\sqrt{1-v^{2}}}\right)^{2}}\right],
$$


which have scales characterized by $\lambda^{-1}$, are singular at $x=v t+x_{0}$, and travel with velocity $-1<v<1$. The energy

$$
E[f]=\int d x\left[\frac{1}{2}\left(\frac{d f}{d x}\right)^{2}+U[f(x)]\right]=\int d x\left[\frac{1}{2}\left(\frac{d f}{d x}\right)^{2}+\frac{m^{2}}{\beta^{2}} \cdot \exp [\beta f(x)]\right]
$$

of these singular solutions is ill-defined, and computed at $x_{0}+v t=0$ is given by the improper integral

$$
E\left[f_{K}\right]=\frac{1}{\lambda \beta^{2}} \int_{-\infty}^{\infty} d x \frac{4}{x^{2}} .
$$

Nevertheless, it is interesting to analyze the small fluctuation spectrum around the singular kink: $\phi(t, x)=f_{K}(x)+\delta \phi(t, x)$. The expansion of the action

$S\left[f_{K}+\delta \phi\right]-S\left[f_{k}\right] \simeq \frac{1}{2} \int d t d x \delta \phi(\lambda t, \lambda x)\left\{-\lambda^{2} \frac{d^{2}}{d x^{2}}+m^{2} \exp \left[\beta f_{k}(x)\right]\right\} \delta \phi(\lambda t, \lambda x)+\mathcal{O}\left(\delta^{3}\right)$

around the singular kink shows that the second order fluctuations are governed by the differential operator

$$
K=-\frac{d^{2}}{d x^{2}}+\frac{d^{2} U}{d \phi^{2}}\left(f_{K}(x)\right)=-\frac{d^{2}}{d x^{2}}+\frac{2}{x^{2}},
$$

which is precisely the Calogero Hamiltonian $H_{1}$ for two particles discussed in the previous section. Moreover, the potential in (3.10) is a first member of the family of Schrödinger operators appearing in de Alfaro-Fubini-Furlan quantum mechanics [29]. The scale invariance $\left(t^{\prime}, x^{\prime}\right)=(\lambda t, \lambda x)$ manifest in the problem of analyzing the kink fluctuations in Liouville conformal field theory, see (3.9), descends this way to a spectral problem in conformal quantum mechanics. We infer this link in the opposite direction as compared to the References $[46,47]$ where an ascending path is travelled from conformal quantum mechanics to Virasoro algebra.

So far we have placed the kink singularity at the origin, $x_{0}+v t=0$, but it is interesting to locate the kink center at the imaginary axis. Setting the scale to unity, $\lambda=1$, and putting the kink at rest, $v=0$, we choose also $x_{0}=-i \alpha, \alpha \in \mathbb{R}$, that is, we displace the kink center from $x_{0}=0$ to the imaginary axis in the complex $x$-plane as a way of escaping the singularity. Either at rest or in motion due to a Lorentz boost the kink profile with its center displaced to the imaginary axis has zero energy:

$$
f_{K}[x, \alpha]=\frac{1}{\beta} \log \left[\frac{2\left(1-v^{2}\right)}{m^{2} \lambda^{2}(x-v t+i \alpha)^{2}}\right], \quad E\left[f_{K}(\alpha]=\frac{1}{\lambda \beta^{2}} \int_{-\infty}^{\infty} d x \frac{4}{(x-v t+i \alpha)^{2}}=0 .\right.
$$

Regarding small fluctuations around these displaced kinks, at rest $v=0$ with unit scale $\lambda=1$, which we write in the $\bmod / \arg$ form,

$$
f_{K}[x, \alpha]=\frac{1}{\beta} \log \left[\frac{2}{m^{2}\left(x^{2}+\alpha^{2}\right)}\right]+\frac{i}{\beta} \arg [i \alpha+x],
$$

the second-order operator becomes:

$$
K^{\alpha}=-\frac{d^{2}}{d x^{2}}+\frac{2}{(x+i \alpha)^{2}}=-\frac{d^{2}}{d x^{2}}+\frac{x^{2}-\alpha^{2}}{\left(x^{2}+\alpha^{2}\right)^{2}}-i \frac{\alpha x}{\left(x^{2}+\alpha^{2}\right)^{2}},
$$


i.e., $K^{\alpha}=H_{1}^{\alpha}$ is the $\mathcal{P} \mathcal{T}$-symmetric Hamiltonian discussed in section 2 .

In order to investigate the spectrum of Liouville kink fluctuations we profit from the Darboux transformation between $H_{0}=D_{1}^{\dagger} D_{1}$ and $H_{1}=D_{1} D_{1}^{\dagger}$. From the plane wave odd eigenfunctions taming the singularity at the origin, in the spectrum of $H_{0}, H_{0} \sin k x=$ $k^{2} \sin k x, k \in \mathbb{R}$, we obtain the eigenfunctions in the continuous spectrum of $H_{1}$ :

$$
H_{1} D_{1}(\sin k x)=D_{1} D_{1}^{\dagger} D_{1}(\sin k x)=k^{2}\left(k \cos k x-\frac{\sin k x}{x}\right) .
$$

In addition, the kink fluctuations include a singular zero mode, $\delta \phi_{0}(x)=\frac{1}{x}$, which is not normalizable because of the singularity at the origin. The kink fluctuations belonging to the continuous spectrum are

$$
\delta \phi_{\omega}(t, x)=e^{i \omega t}\left(\frac{d}{d x}-\frac{1}{x}\right) \sin k x=e^{i \omega t}\left(k \cos k x-\frac{\sin k x}{x}\right), \quad \omega=k .
$$

We again stress that the scale invariance of the Liouville field theory descends to scale invariance of the conformal quantum mechanics that governs the Liouville kink fluctuations. The dispersion relation $\omega=k$ tells us that kink fluctuations are "massless" as a consequence of $(1+1)$-dimensional conformal symmetry. Comparing the $H_{0}$ and $H_{1}$ eigenfunctions at long distances, $\sin (k x) \propto e^{i k x}-e^{-i k x}$ versus $\cos (k x) \propto e^{i k x}+e^{-i k x}$, we observe that a phase $-1=e^{i \pi}, k$-independent, arises for the reflected waves in the $n=1$ Calogero potential non-existing in the free motion of one particle on the right half-line with Dirichlet boundary conditions, see appendix. This phase can be interpreted from the well studied fermionic character of kinks and solitons.

In the case of the kinks displaced into the imaginary axis things are identical with respect to small fluctuations except that now the Darboux-related operators

$$
H_{0}=D_{1}^{\#} D_{1}=-\frac{d^{2}}{d x^{2}} \quad \text { and } \quad H_{1}^{\alpha}=D_{1} D_{1}^{\#}=-\frac{d^{2}}{d x^{2}}+\frac{2}{(x+i \alpha)^{2}}
$$

are intertwined by the first-order operators $D_{1}=\frac{d}{d x}-\frac{1}{x+i \alpha}$ and $D_{1}^{\#}=-\frac{d}{d x}-\frac{1}{x+i \alpha}$. Starting from the odd eigenfunctions of the free particle we find through the action of $D_{1}$ the continuous spectrum eigenfunctions of $K_{1}^{\alpha}$ :

$$
\delta \phi_{\omega}(t, x)=e^{i \omega t}\left(\frac{d}{d x}-\frac{1}{x+i \alpha}\right) \sin k x=e^{i \omega t}\left(k \cos k x-\frac{x-i \alpha}{x^{2}+\alpha^{2}} \sin k x\right), \quad \omega=k .
$$

In this case the real part of these eigenfunctions is non-null at the origin, $\operatorname{Re} \delta \phi_{\omega}(0,0)=k$, but the imaginary part vanishes at $(t=0, x=0): \operatorname{Im} \delta \phi_{\omega}(0,0)=0$. Besides the continuous spectrum eigenfunctions, there is a kink zero mode of fluctuation annihilated by $D_{1}^{\#}$,

$$
\frac{d \delta \phi_{0}}{d x}+\frac{1}{x+i \alpha} \delta \phi_{0}(x)=0 \Rightarrow \delta \phi_{0}(x)=\frac{1}{x+i \alpha}
$$

which is regular and quadratically integrable on real line, but is purely imaginary at $x=0$. 


\subsection{Kinks in the SU(3) conformal Toda model}

The SU(3) conformal Toda field theory [50] encompasses two $(1+1)$-dimensional scalar fields assembled into a vector field through use of the simple roots of the SU(3) Lie algebra: $\vec{\alpha}_{1} \cdot \vec{\alpha}_{1}=\vec{\alpha}_{2} \cdot \vec{\alpha}_{2}=2, \vec{\alpha}_{1} \cdot \vec{\alpha}_{2}=\vec{\alpha}_{2} \cdot \vec{\alpha}_{1}=-1$,

$$
\vec{\psi}(t, x)=\vec{\alpha}_{1} \psi_{1}(t, x)+\vec{\alpha}_{2} \psi_{2}(t, x), \quad C=2\left(\begin{array}{cc}
\frac{\vec{\alpha}_{1} \cdot \vec{\alpha}_{1}}{\left|\vec{\alpha}_{1}\right| \cdot\left|\vec{\alpha}_{1}\right|} & \frac{\vec{\alpha}_{1} \cdot \vec{\alpha}_{2}}{\left|\vec{\alpha}_{1}\right| \cdot\left|\vec{\alpha}_{2}\right|} \\
\frac{\vec{\alpha}_{2} \cdot \vec{\alpha}_{1}}{\left|\vec{\alpha}_{2}\right| \cdot\left|\vec{\alpha}_{1}\right|} & \frac{\vec{\alpha}_{2} \cdot \vec{\alpha}_{2}}{\left|\vec{\alpha}_{2}\right| \cdot\left|\vec{\alpha}_{2}\right|}
\end{array}\right)=\left(\begin{array}{cc}
2 & -1 \\
-1 & 2
\end{array}\right),
$$

where $C$ is the $\mathrm{SU}(3)$ Cartan matrix. The conformal Toda Lagrangian is

$$
\mathcal{L}[\vec{\psi}]=\frac{1}{2} \frac{\partial \vec{\psi}}{\partial t} \cdot \frac{\partial \vec{\psi}}{\partial t}-\frac{1}{2} \frac{\partial \vec{\psi}}{\partial x} \cdot \frac{\partial \vec{\psi}}{\partial x}-\frac{m^{2}}{\beta^{2}}\left[\exp \left[\frac{\beta}{\sqrt{2}} \vec{\alpha}_{1} \cdot \vec{\psi}(t, x)\right]+\exp \left[\frac{\beta}{\sqrt{2}} \vec{\alpha}_{2} \cdot \vec{\psi}(t, x)\right]\right]
$$

and the field equations read

$$
\begin{aligned}
& 4 \frac{\partial^{2} \psi_{1}}{\partial x_{+} \partial x_{-}}-2 \frac{\partial^{2} \psi_{2}}{\partial x_{+} \partial x_{-}}+\frac{m^{2}}{\beta}\left[2 \exp \left[\frac{\beta}{\sqrt{2}}\left(2 \psi_{1}-\psi_{2}\right)\right]-\exp \left[\frac{\beta}{\sqrt{2}}\left(2 \psi_{2}-\psi_{1}\right)\right]\right]=0, \\
& 4 \frac{\partial^{2} \psi_{2}}{\partial x_{+} \partial x_{-}}-2 \frac{\partial^{2} \psi_{1}}{\partial x_{+} \partial x_{-}}+\frac{m^{2}}{\beta}\left[2 \exp \left[\frac{\beta}{\sqrt{2}}\left(2 \psi_{2}-\psi_{1}\right)\right]-\exp \left[\frac{\beta}{\sqrt{2}}\left(2 \psi_{1}-\psi_{2}\right)\right]\right]=0
\end{aligned}
$$

The PDE system (3.18), (3.19) is manifestly conformal invariant if the space-time transformations $x_{ \pm}^{\prime}=f_{ \pm}\left(x_{ \pm}\right)$are accompanied by the field redefinitions:

$$
\begin{aligned}
& \psi_{1}^{\prime}\left(x_{+}^{\prime}, x_{-}^{\prime}\right)=-\frac{\sqrt{2}}{\beta} \ln \left[\partial_{+} f_{+}\left(x_{+}\right) \partial_{-} f_{-}\left(x_{-}\right)\right]+\psi_{1}\left(x_{+}, x_{-}\right), \\
& \psi_{2}^{\prime}\left(x_{+}^{\prime}, x_{-}^{\prime}\right)=-\frac{\sqrt{2}}{\beta} \ln \left[\partial_{+} f_{+}\left(x_{+}\right) \partial_{-} f_{-}\left(x_{-}\right)\right]+\psi_{2}\left(x_{+}, x_{-}\right) .
\end{aligned}
$$

Since the eigenvectors of the Cartan matrix $C$ are $v_{1}=\left(\begin{array}{l}1 \\ 1\end{array}\right)$ and $v_{2}=\left(\begin{array}{c}1 \\ -1\end{array}\right)$, with eigenvalues 1 and 3, respectively, it is convenient to perform the following change of variables in the fields : $\phi_{1}=\frac{1}{\sqrt{2}}\left(\psi_{1}+\frac{1}{\sqrt{3}} \psi_{2}\right)$ and $\phi_{2}=\frac{1}{\sqrt{2}}\left(\psi_{1}-\frac{1}{\sqrt{3}} \psi_{2}\right)$. In the new fields the $\mathrm{SU}(3)$ conformal Toda field theory action becomes:

$$
\begin{aligned}
S\left[\phi_{1}, \phi_{2}\right] & =\iint d t d x\left\{\frac{1}{2}\left(\frac{\partial \phi_{1}}{\partial t}\right)^{2}+\frac{1}{2}\left(\frac{\partial \phi_{2}}{\partial t}\right)^{2}-\frac{1}{2}\left(\frac{\partial \phi_{1}}{\partial x}\right)^{2}-\frac{1}{2}\left(\frac{\partial \phi_{2}}{\partial x}\right)^{2}-U\left(\phi_{1}, \phi_{2}\right)\right\}, \\
U\left(\phi_{1}, \phi_{2}\right) & =\frac{m^{2}}{\beta^{2}} \cdot\left\{\exp \left[\frac{\beta}{2}\left(\phi_{1}(t, x)+\sqrt{3} \phi_{2}(t, x)\right)\right]+\exp \left[\frac{\beta}{2}\left(\phi_{1}(t, x)-\sqrt{3} \phi_{2}(t, x)\right)\right]\right\},
\end{aligned}
$$

and the Toda field equations are diagonal in the field derivatives:

$$
\begin{aligned}
\frac{\partial^{2} \phi_{1}}{\partial t^{2}}(t, x)-\frac{\partial^{2} \phi_{1}}{\partial x^{2}}(t, x)+\frac{m^{2}}{2 \beta}\left(e^{\frac{\beta}{2}\left(\phi_{1}(t, x)+\sqrt{3} \phi_{2}(t, x)\right)}+e^{\frac{\beta}{2}\left(\phi_{1}(t, x)-\sqrt{3} \phi_{2}(t, x)\right)}\right) & =0, \\
\frac{\partial^{2} \phi_{2}}{\partial t^{2}}(t, x)-\frac{\partial^{2} \phi_{2}}{\partial x^{2}}(t, x)+\frac{\sqrt{3} m^{2}}{2 \beta}\left(e^{\frac{\beta}{2}\left(\phi_{1}(t, x)+\sqrt{3} \phi_{2}(t, x)\right)}-e^{\frac{\beta}{2}\left(\phi_{1}(t, x)-\sqrt{3} \phi_{2}(t, x)\right)}\right) & =0 .
\end{aligned}
$$


Like the Liouville PDE this system of PDE's is completely integrable in terms of arbitrary functions of $x_{+}$and $x_{-}$with the help of the infinite dimensional conformal symmetry with Lie algebra being some variant of Kac-Moody algebra.

The second-order variations around any solution $\left(\phi_{1}^{S}(t, x), \phi_{2}^{S}(t, x)\right)$ of these PDE system are governed by the Hessian operator

$$
\mathcal{H}=\left(\begin{array}{cc}
\frac{\partial^{2}}{\partial t^{2}}-\frac{\partial^{2}}{\partial x^{2}}+\frac{\delta^{2} U}{\delta \phi_{1}^{2}}\left(\phi_{1}^{S}(t, x), \phi_{2}^{S}(t, x)\right) & \frac{\delta^{2} U}{\delta \phi_{1} \delta \phi_{2}}\left(\phi_{1}^{S}(t, x), \phi_{2}^{S}(t, x)\right) \\
\frac{\delta^{2} U}{\delta \phi_{2} \delta \phi_{1}}\left(\phi_{1}^{S}(t, x), \phi_{2}^{S}(t, x)\right) & \frac{\partial^{2}}{\partial t^{2}}-\frac{\partial^{2}}{\partial x^{2}}+\frac{\delta^{2} U}{\delta \phi_{2}^{2}}\left(\phi_{1}^{S}(t, x), \phi_{2}^{S}(t, x)\right)
\end{array}\right)
$$

where

$$
\begin{aligned}
\frac{\delta^{2} U}{\delta \phi_{1}^{2}}\left(\phi_{1}^{S}(t, x), \phi_{2}^{S}(t, x)\right) & =\frac{m^{2}}{4}\left[e^{\frac{\beta}{2}\left(\phi_{1}^{S}(t, x)+\sqrt{3} \phi_{2}^{S}(t, x)\right)}+e^{\frac{\beta}{2}\left(\phi_{1}^{S}(t, x)-\sqrt{3} \phi_{2}^{S}(t, x)\right)}\right], \\
\frac{\delta^{2} U}{\delta \phi_{1} \delta \phi_{2}}\left(\phi_{1}^{S}(t, x), \phi_{2}^{S}(t, x)\right) & =\frac{\sqrt{3} m^{2}}{4}\left[e^{\frac{\beta}{2}\left(\phi_{1}^{S}(t, x)+\sqrt{3} \phi_{2}^{S}(t, x)\right)}-e^{\left.\frac{\beta}{2}\left(\phi_{1}^{S}(t, x)-\sqrt{3} \phi_{2}^{S}(t, x)\right)\right],}\right. \\
\frac{\delta^{2} U}{\delta \phi_{2} \delta \phi_{1}}\left(\phi_{1}^{S}(t, x), \phi_{2}^{S}(t, x)\right) & =\frac{\sqrt{3} m^{2}}{4}\left[e^{\frac{\beta}{2}\left(\phi_{1}^{S}(t, x)+\sqrt{3} \phi_{2}^{S}(t, x)\right)}-e^{\left.\frac{\beta}{2}\left(\phi_{1}^{S}(t, x)-\sqrt{3} \phi_{2}^{S}(t, x)\right)\right],}\right. \\
\frac{\delta^{2} U}{\delta \phi_{2}^{2}}\left(\phi_{1}^{S}(t, x), \phi_{2}^{S}(t, x)\right) & =\frac{3 m^{2}}{4}\left[e^{\frac{\beta}{2}\left(\phi_{1}^{S}(t, x)+\sqrt{3} \phi_{2}^{S}(t, x)\right)}+e^{\frac{\beta}{2}\left(\phi_{1}^{S}(t, x)-\sqrt{3} \phi_{2}^{S}(t, x)\right)}\right] .
\end{aligned}
$$

We first consider the only solution of the PDE system (3.20), (3.21) independent of $t$ and $x:\left(\phi_{1}^{0}=-\infty, \phi_{2}^{0}=0\right)$, which is the absolute minimum of $U\left(\phi_{1}, \phi_{2}\right)$. Clearly, the Hessian evaluated at this constant solution is $\mathcal{H}^{0}=\left(\begin{array}{cc}\frac{\partial^{2}}{\partial t^{2}}-\frac{\partial^{2}}{\partial x^{2}} & 0 \\ 0 & \frac{\partial^{2}}{\partial t^{2}}-\frac{\partial^{2}}{\partial x^{2}}\end{array}\right)$. Since the spectrum of $\mathcal{H}^{0}$ is positive, the solution $\left(\phi_{1}^{0}, \phi_{2}^{0}\right)$ is classicaly stable with respect to small fluctuations. Thus, in the quantum framework these fluctuations of "vacuum" become massless bosons (compatible with conformal symmetry).

The next step is the search for solitary wave type of solutions, that is, making the ansatz $\left(\phi_{1}(t, x)=f(x), \phi_{2}(t, x)=0\right)$ (in the kink center of mass), the PDE system above reduces to the first-order ODE

$$
\frac{d f}{d x}=2 \frac{m}{\beta} \cdot \exp \left[\frac{\beta}{4} f(x)\right] .
$$

Setting the kink scale to unit, $\lambda=1$, Lorentz and translation invariance provide the family of singular kinks

$$
f_{K}(t, x)=\frac{2}{\beta} \log \left[\frac{4\left(1-v^{2}\right)}{m^{2}\left(x-v t-x_{0}\right)}\right]
$$

traveling with velocity $-1<v<1$. The energy $E[(f, 0)]=$ $\int d x\left[\frac{1}{2}\left(\frac{d f}{d x}\right)^{2}+2 \frac{m^{2}}{\beta^{2}} \cdot \exp \left[\frac{\beta}{2} f(x)\right]\right]$ of these singular solutions is ill defined. Computed at $x_{0}=0$ it is given by the improper integral

$$
E\left[\left(f_{K}, 0\right)\right]=\frac{1}{\beta^{2}} \int_{-\infty}^{\infty} d x \frac{4}{x^{2}} .
$$


Like in the case of the Liouville kinks it is interesting to analyze the small fluctuation spectrum around these Toda singular kinks: $\left(\phi_{1}(t, x)=f_{K}(x)+\delta \phi_{1}(t, x), \phi_{2}(t, x)=\delta \phi_{2}(t, x)\right)$. The fluctuations up to second-order $\mathcal{O}\left(\delta^{2}\right)$ belong to the kernel of $\mathcal{H}\left(f_{K}(x), 0\right)$ :

$$
\mathcal{H}\left(f_{K}(x), 0\right)=\left(\begin{array}{cc}
\frac{\partial^{2}}{\partial t^{2}} & 0 \\
0 & \frac{\partial^{2}}{\partial t^{2}}
\end{array}\right)+K=\left(\begin{array}{cc}
\frac{\partial^{2}}{\partial t^{2}} & 0 \\
0 & \frac{\partial^{2}}{\partial t^{2}}
\end{array}\right)+\left(\begin{array}{cc}
-\frac{\partial^{2}}{\partial x^{2}}+\frac{2}{x^{2}} & 0 \\
0 & -\frac{\partial^{2}}{\partial x^{2}}+\frac{6}{x^{2}}
\end{array}\right) .
$$

The Toda kink fluctuations thus can be expanded in terms of the eigenfunctions of the matrix differential operator $K$ :

$$
K\left(\begin{array}{l}
\delta \phi_{1}^{\omega}(x) \\
\delta \phi_{2}^{\omega}(x)
\end{array}\right)=\left(\begin{array}{cc}
-\frac{d^{2}}{d x^{2}}+\frac{2}{x^{2}} & 0 \\
0 & -\frac{d^{2}}{d x^{2}}+\frac{6}{x^{2}}
\end{array}\right)\left(\begin{array}{l}
\delta \phi_{1}^{\omega}(x) \\
\delta \phi_{2}^{\omega}(x)
\end{array}\right)=\omega^{2}\left(\begin{array}{c}
\delta \phi_{1}^{\omega}(x) \\
\delta \phi_{2}^{\omega}(x)
\end{array}\right) .
$$

From the explicit form of the hessian (3.30) we clearly see that its kernel $\mathcal{H} \delta \phi(t, x)$ is Fourier expanded in terms of the K-eigenfunctions:

$$
\mathcal{H} \delta \phi(t, x)=0 \Leftrightarrow \delta \phi(t, x)=\int_{-\infty}^{\infty} d \omega e^{i \omega t}\left(\begin{array}{l}
\delta \phi_{1}^{\omega}(x) \\
\delta \phi_{2}^{\omega}(x)
\end{array}\right) .
$$

Since $K$ is a diagonal matrix of two-particle Calogero Hamiltonians, the conformal Toda kink fluctuations exhibit similar features to the properties of Liouville kink fluctuations just analyzed in the previous sub-section. In particular, the fluctuations in the $\phi_{1}$ field, $\delta \phi_{1}^{\omega}(x)$, are exactly the eigenfunctions in the continuous spectrum of $H_{1}$ vanishing at $x=0$. As for Liouville kinks it is possible to escape from the singularity at $x=0$ by sending the kink center to the imaginary axis.

The novelties arise in the $\delta \phi_{2}(t, x)$ fluctuations. These fluctuations may be expanded in terms of the eigenfunctions of $H_{2}$, the $n=2$ two-particle Calogero Hamiltonian $\mathrm{H}_{2}$ :

$$
\delta \phi_{2}^{\omega}(x)=\left(\frac{d}{d x}-\frac{2}{x}\right)\left(\frac{d}{d x}-\frac{1}{x}\right) \sin k x=-\frac{3 k}{x} \cos k x+\frac{3-k^{2} x^{2}}{x^{2}} \sin k x, \quad \omega^{2}=k^{2} .
$$

This is possible because the hierarchy

$$
\begin{aligned}
H_{0}=D_{1}^{\dagger} D_{1}, & H_{1}=D_{1} D_{1}^{\dagger}, \\
H_{1} & =D_{2}^{\dagger} D_{2}, \quad H_{2}=D_{2} D_{2}^{\dagger}
\end{aligned}
$$

allows us to obtain the eigenfunctions of $H_{2}$ iteratively from the $H_{0}$ eigenfunctions:

$$
H_{2}\left(-\frac{3 k}{x} \cos k x+\frac{3-k^{2} x^{2}}{x^{2}} \sin k x\right)=k^{2}\left(-\frac{3 k}{x} \cos k x+\frac{3-k^{2} x^{2}}{x^{2}} \sin k x\right) .
$$

Besides the positive eigenfunctions, the spectrum of $\mathrm{H}_{2}$ includes a singular zero mode:

$$
D_{2}^{\dagger} \delta \phi_{2}^{0}(x)=0 \Rightarrow \delta \phi_{2}^{0}(x) \propto \frac{1}{x^{2}}
$$

to be added to the kink translational mode: $\delta \phi_{1}^{0}(x) \propto \frac{1}{x}$. 
We omit the description of these singular Toda kinks regularized by pushing their centers to the imaginary axis because their properties are essentially identical to those of regular Liouville kinks. We conclude this section by mentioning that the one-loop mass shifts of Liouville and Toda kinks are null. Since the kink Hessians are reflectionless, the Cahill-Comtet-Glauber formula [51-53] can be applied. Because the discrete and continuous spectra collapse at the point $k=0$, the threshold of the continuous spectrum, the CCG formula gives mass shifts equal to zero. This is because the CCG formula expresses the one-loop kink mass shift as a sum of terms collecting the bound state contributions times the scattering threshold that in this case is zero. The annihilation of one-loop kink mass shifts is thus the consequence of perfect invisibility of the Calogero Hamiltonians.

\section{Exotic supersymmetry of the pairs of perfectly invisible $\mathcal{P} \mathcal{T}$ - symmetric systems}

In this section we investigate the exotic nonlinear supersymmetry structure appearing in the extended systems composed from the pairs of perfectly invisible $\mathcal{P} \mathcal{T}$-symmetric quantum systems related by the first order Darboux transformations.

Consider the simplest extended system composed from the pair $H_{0}$ and $H_{1}^{\alpha}$ and described by the matrix Hamiltonian

$$
\mathcal{H}=\left(\begin{array}{cc}
H_{1}^{\alpha} & 0 \\
0 & H_{0}
\end{array}\right) .
$$

The extended system has the integrals of motion

$$
\begin{aligned}
Q_{1} & =\left(\begin{array}{cc}
0 & D_{1} \\
D_{1}^{\#} & 0
\end{array}\right), & Q_{2}=i \sigma_{3} Q_{1} \\
S_{1} & =\left(\begin{array}{cc}
0 & -i D_{1} \mathcal{P}_{0} \\
i \mathcal{P}_{0} D_{1}^{\#} & 0
\end{array}\right), & S_{2}=i \sigma_{3} S_{1}
\end{aligned}
$$

where $D_{1}$ is the first order differential operator given by eq. (2.8), and $\mathcal{P}_{0}=-i \frac{d}{d x}$ is the momentum operator of the free particle $H_{0}$. Operators $Q_{a}$ and $S_{a}, a=1,2$, constitute the set of four supercharges of the extended system: they commute with the Hamiltonian $\mathcal{H}$,

$$
\left[\mathcal{H}, Q_{a}\right]=0, \quad\left[\mathcal{H}, S_{a}\right]=0,
$$

and their anticommutation relations are

$$
\begin{aligned}
\left\{Q_{a}, Q_{b}\right\} & =2 \delta_{a b} \mathcal{H}, \quad\left\{S_{a}, S_{b}\right\}=2 \delta_{a b} \mathcal{H}^{2}, \\
\left\{Q_{a}, S_{b}\right\} & =2 \epsilon_{a b} \mathcal{L}_{1} .
\end{aligned}
$$

Here

$$
\mathcal{L}_{1}=\left(\begin{array}{cc}
\mathcal{P}_{1}^{\alpha} & 0 \\
0 & H_{0} \mathcal{P}_{0}
\end{array}\right)
$$


is the bosonic integral of motion composed from the Lax-Novikov integrals of motion of the subsystems $H_{1}^{\alpha}$ and $H_{0}$. One also can define a bosonic operator $\mathcal{L}_{2}=\sigma_{3} \mathcal{L}_{1}$. It is another nontrivial bosonic integral of the system,

$$
\left[\mathcal{H}, \mathcal{L}_{a}\right]=0
$$

$a=1,2$. We have the following commutation relations between bosonic, $\mathcal{L}_{a}$, and fermionic, $Q_{a}$ and $S_{a}$, integrals of motion of the system $\mathcal{H}$ :

$$
\begin{array}{lll}
{\left[\mathcal{L}_{1}, Q_{a}\right]=0,} & & {\left[\mathcal{L}_{1}, S_{a}\right]=0,} \\
{\left[\mathcal{L}_{2}, Q_{a}\right]=2 i \mathcal{H} S_{a},} & & {\left[\mathcal{L}_{2}, S_{a}\right]=-2 i \mathcal{H}^{2} Q_{a} .}
\end{array}
$$

The set of (anti)-commutation relations (4.4), (4.5), (4.6), (4.8), (4.9) and (4.10) is the exotic nonlinear superalgebra of the system (4.1). The Lie superalgebra of conventional $\mathcal{N}=2$ supersymmetry is contained here as a sub-superalgebra generated by the Hamiltonian $\mathcal{H}$ and the first order supercharges $Q_{a}$. The nonlinear extension of superalgebra emerges here because the subsystem $H_{0}$ has its proper integral of motion $\mathcal{P}_{0}=-i \frac{d}{d x}$ that enters the structure of the pair of additional supercharges $S_{a}$ which are matrix differential operators of order two. Like the Hamiltonian operator $\mathcal{H}$, the bosonic integral $\mathcal{L}_{1}$ composed from the Lax-Novikov integrals of motion of the subsystems is a central element of the superalgebra. The second bosonic integral $\mathcal{L}_{2}$ when commutes with the pair of supercharges $Q_{a}\left(S_{a}\right)$, generates another pair of supercharges $S_{a}\left(Q_{a}\right)$. The nonlinearity of the superalgebra is related to the appearance of the central charge $\mathcal{H}$ in the form of multipliers $\mathcal{H}$ and $\mathcal{H}^{2}$ in the anticommutator of supercharges $S_{a}$ and in commutation relations (4.10) of the supercharges with the bosonic integral $\mathcal{L}_{2}$.

The supersymmetry of the extended system described by a Hamiltonian $\mathcal{H}=$ $\operatorname{diag}\left(H_{n}^{\alpha}, H_{n-1}^{\alpha}\right)$ composed from a pair of perfectly invisible neighbour $\mathcal{P} \mathcal{T}$-symmetric systems from the family (2.16) has a similar structure. Two supercharges $Q_{a}$ are matrix differential operators of the first order which have the form similar to (4.2) with operators $D_{1}$ and $D_{1}^{\#}$ changed for the operators $D_{n}$ and $D_{n}^{\#}$ defined in (2.19). The supercharges $S_{a}$ are matrix differential operators of order $2 n$. They have a structure similar to that of the supercharges (4.3) with the operators $D_{1} \mathcal{P}_{0}$ and $\mathcal{P}_{0} D_{1}^{\#}$ changed for $D_{n} \mathcal{P}_{n-1}^{\alpha}$ and $\mathcal{P}_{n-1}^{\alpha} D_{n}^{\#}$, respectively, where $\mathcal{P}_{n-1}^{\alpha}$ is the Lax-Novikov integral of the subsystem $H_{n-1}^{\alpha}$ given by eq. (2.23). The analog of the bosonic integral (4.7) has in this case the form $\mathcal{L}_{1}=\operatorname{diag}\left(\mathcal{P}_{n}^{\alpha}, H_{n-1}^{\alpha} \mathcal{P}_{n-1}^{\alpha}\right)$, and, again, another nontrivial bosonic integral is $\mathcal{L}_{2}=\sigma_{3} \mathcal{L}_{1}$. The anti-commutator of supercharges $Q_{a}$ with $Q_{b}$ has the same form as in (4.5), the anticommutator of $Q_{a}$ with $S_{b}$ has the form (4.6). The anti-commutator between supercharges $S_{a}$ and $S_{b}$ changes here for $\left\{S_{a}, S_{b}\right\}=2 \delta_{a b}(\mathcal{H})^{2 n}$, which is a corresponding generalization of the second relation from (4.5). Here $\mathcal{L}_{1}$ is again a central element of superalgebra generated via the anti-commutator between supercharges $Q_{a}$ and $S_{b}$. The commutator of $\mathcal{L}_{2}$ with $Q_{a}$ has exactly the same form as in (4.10), while the commutator $\mathcal{L}_{2}$ with $S_{a}$ is changed for $\left[\mathcal{L}_{2}, S_{a}\right]=-2 i \mathcal{H}^{2 n} Q_{a}$, that is a natural generalization of the second relation from (4.10). Note that the matrix differential operator $K$ from eq. (3.31) that controls the Toda kink fluctuations, after $\mathcal{P} \mathcal{T}$-regularization $x \rightarrow x+i \alpha$ corresponds exactly to the case $n=2$ 
of the supersymmetric Hamiltonian $\mathcal{H}=\operatorname{diag}\left(H_{n}^{\alpha}, H_{n-1}^{\alpha}\right)$ with permuted subsystems. So, the system of $\mathcal{P} \mathcal{T}$-regularized kinks of the $\mathrm{SU}(3)$ conformal Toda system is described by the exotic $\mathcal{N}=4$ nonlinear supersymmetry.

In the same way one can identify the superalgebra of the extended systems composed from the pairs of the systems $H_{\alpha, \gamma}^{(1)}$ and $H_{1}^{\alpha}$, and of the systems $H_{\alpha, \gamma}^{(2)}$ and $H_{2}^{\alpha}$, where $H_{\alpha, \gamma}^{(1)}$ and $H_{\alpha, \gamma}^{(2)}$ are the perfectly invisible $\mathcal{P} \mathcal{T}$-symmeric systems that we described at the end of section 2 .

We also can consider the exotic supersymmetry of an extended system composed from any two perfectly invisible $\mathcal{P} \mathcal{T}$-symmetric systems by taking into account that each such a system is characterized by a nontrivial Lax-Novikov integral being a Darboux-dressed momentum operator of the free particle. As a consequence, the corresponding two subsystems always can be intertwined by two distinct differential operators, see for such a structure appearing in $n$-soliton [54] and periodic finite-gap [55] systems.

Like in the case of reflectionless systems $[54,56,57]$, sometimes there could appear the operators which intertwine the two perfectly invisible $\mathcal{P} \mathcal{T}$-symmeric systems directly but not via the chains of the intertwining operators ascending to the free particle system. Then the structure of the superymmetry algebra will transmute and include some central element composed from the complex shift parameters.

As an example where such a situation is realized, let us consider the extended system

$$
\mathcal{H}=\left(\begin{array}{cc}
H_{1}^{\alpha_{2}} & 0 \\
0 & H_{1}^{\alpha_{1}}
\end{array}\right)
$$

composed from two systems $H_{1}^{\alpha_{1}}$ and $H_{1}^{\alpha_{2}}$ of the form (2.9) characterized by two different nonzero shift parameters $\alpha_{1}$ and $\alpha_{2}$. For the sake of definiteness, we assume that $\alpha_{1}>\alpha_{2}$. The two subsystems $H_{1}^{\alpha_{1}}$ and $H_{1}^{\alpha_{2}}$ can be intertwined by the second order differential operators $D_{\alpha_{1}} D_{\alpha_{2}}^{\#}$ and $D_{\alpha_{2}} D_{\alpha_{1}}^{\#}$, where $D_{\alpha_{j}}$ and $D_{\alpha_{j}}^{\#}, j=1,2$, are the first order differential operators of the form (2.8), $D_{\alpha_{j}}=\frac{d}{d x}-\left(x+i \alpha_{j}\right)^{-1}, D_{\alpha_{j}}^{\#}=-\frac{d}{d x}-\left(x+i \alpha_{j}\right)^{-1}$, each of which intertwines a subsystem $H_{1}^{\alpha_{j}}$ with a free particle $H_{0}$, see eq. (2.10). So, we have here the intertwining relations

$$
\left(D_{\alpha_{1}} D_{\alpha_{2}}^{\#}\right) H_{1}^{\alpha_{2}}=H_{1}^{\alpha_{1}}\left(D_{\alpha_{1}} D_{\alpha_{2}}^{\#}\right), \quad\left(D_{\alpha_{2}} D_{\alpha_{1}}^{\#}\right) H_{1}^{\alpha_{1}}=H_{1}^{\alpha_{2}}\left(D_{\alpha_{2}} D_{\alpha_{1}}^{\#}\right) .
$$

These second order differential operators intertwine the subsystems $H_{1}^{\alpha_{1}}$ and $H_{1}^{\alpha_{2}}$ effectively via a 'virtual' free particle system $H_{0}:\left(D_{\alpha_{1}} D_{\alpha_{2}}^{\#}\right) H_{1}^{\alpha_{2}}=D_{\alpha_{1}}\left(D_{\alpha_{2}}^{\#} H_{1}^{\alpha_{2}}\right)=\left(D_{\alpha_{1}} H_{0}\right) D_{\alpha_{2}}^{\#}=$ $H_{1}^{\alpha_{1}}\left(D_{\alpha_{1}} D_{\alpha_{2}}^{\#}\right)$. Besides them, we also have here the intertwiners

$$
D=\frac{d}{d x}+\mathcal{W}, \quad D^{\#}=-\frac{d}{d x}+\mathcal{W}
$$

where

$$
\mathcal{W}=\frac{1}{\xi_{1}}-\frac{1}{\xi_{2}}-\frac{1}{\xi_{1}-\xi_{2}},
$$

$\xi_{j}=x+i \alpha_{j}$, and $-\left(\xi_{1}-\xi_{2}\right)^{-1}=i\left(\alpha_{1}-\alpha_{2}\right)^{-1}$. The first order differential operators (4.13) intertwine the subsystems directly,

$$
D H_{1}^{\alpha_{1}}=H_{1}^{\alpha_{2}} D, \quad D^{\#} H_{1}^{\alpha_{2}}=H_{1}^{\alpha_{1}} D^{\#},
$$


and also satisfy the relations

$$
D^{\#} D=H_{1}^{\alpha_{1}}-\Delta^{2}, \quad D D^{\#}=H_{1}^{\alpha_{2}}-\Delta^{2},
$$

where we have introduced the notation

$$
\Delta=\frac{1}{\alpha_{1}-\alpha_{2}} .
$$

When $\alpha_{1} \rightarrow \infty$, the Hamiltonian operator $H_{1}^{\alpha_{1}}$ transforms into $H_{0}$, for function (4.14) we have $\mathcal{W} \rightarrow-\xi_{2}^{-1}$, and $D$ transforms into the operator $D_{1}$ given by eq. (2.8) with $\xi$ changed for $\xi_{2}$, that intertwines the $H_{0}$ with $H_{1}^{\alpha_{2}}$.

To establish the explicit form of the exotic superalgebraic structure of the system (4.11), the following relations that involve the operators (4.13), and $D_{\alpha_{j}}$ and $D_{\alpha_{j}}^{\#}$, $j=1,2$, are useful :

$$
\begin{aligned}
D D_{\alpha_{1}} & =D_{\alpha_{2}} D_{0}, & D_{\alpha_{2}}^{\#} D & =D_{0} D_{\alpha_{1}}^{\#}, \\
D^{\#} D_{\alpha_{2}} & =D_{\alpha_{1}} D_{0}^{\#}, & D_{\alpha_{1}}^{\#} D^{\#} & =D_{0}^{\#} D_{\alpha_{2}}^{\#},
\end{aligned}
$$

where we denoted

$$
D_{0} \equiv \frac{d}{d x}+i \Delta, \quad D_{0}^{\#} \equiv-\frac{d}{d x}+i \Delta
$$

Note that the first order operators (4.13) and the second order operators $D_{\alpha_{1}} D_{\alpha_{2}}^{\#}$ and $D_{\alpha_{2}} D_{\alpha_{1}}^{\#}$ intertwine also the Lax-Novikov integrals of the subsystems,

$$
\begin{aligned}
D \mathcal{P}^{\alpha_{1}} & =\mathcal{P}^{\alpha_{2}} D, & D^{\#} \mathcal{P}^{\alpha_{2}} & =\mathcal{P}^{\alpha_{1}} D^{\#} \\
\left(D_{\alpha_{2}} D_{\alpha_{1}}^{\#}\right) \mathcal{P}^{\alpha_{1}} & =\mathcal{P}^{\alpha_{2}}\left(D_{\alpha_{2}} D_{\alpha_{1}}^{\#}\right), & \left(D_{\alpha_{1}} D_{\alpha_{2}}^{\#}\right) \mathcal{P}^{\alpha_{2}} & =\mathcal{P}^{\alpha_{1}}\left(D_{\alpha_{1}} D_{\alpha_{2}}^{\#}\right) .
\end{aligned}
$$

From the described intertwining relations we find that the system (4.11) is characterized by the supercharges

$$
Q_{1}=\left(\begin{array}{cc}
0 & D \\
D^{\#} & 0
\end{array}\right), \quad Q_{2}=i \sigma_{3} Q_{1},
$$

which are the matrix first order differential operators, and by the supercharges

$$
S_{1}=\left(\begin{array}{cc}
0 & D_{\alpha_{2}} D_{\alpha_{1}}^{\#} \\
D_{\alpha_{1}} D_{\alpha_{2}}^{\#} & 0
\end{array}\right), \quad S_{2}=i \sigma_{3} S_{1}
$$

which are matrix differential operators of the second order. Besides, the extended system (4.11) has two nontrivial bosonic integrals of motion

$$
\mathcal{L}_{1}=\left(\begin{array}{cc}
\mathcal{P}^{\alpha_{2}} & 0 \\
0 & \mathcal{P}^{\alpha_{1}}
\end{array}\right), \quad \mathcal{L}_{2}=\sigma_{3} \mathcal{L}_{1}
$$


All these integrals of motion generate the following exotic nonlinear superalgebra of the system (4.11):

$$
\begin{aligned}
& {\left[\mathcal{H}, Q_{a}\right]=0,} \\
& \left\{Q_{a}, Q_{b}\right\}=2 \delta_{a b}\left(\mathcal{H}-\Delta^{2}\right), \\
& {\left[\mathcal{H}, S_{a}\right]=0, \quad\left[\mathcal{H}, \mathcal{L}_{a}\right]=0,} \\
& \left\{Q_{a}, S_{b}\right\}=2\left(\epsilon_{a b} \mathcal{L}_{1}+i \delta_{a b} \Delta \mathcal{H}\right) \text {, } \\
& \left\{S_{a}, S_{b}\right\}=2 \delta_{a b} \mathcal{H}^{2} \\
& {\left[\mathcal{L}_{a}, \mathcal{L}_{b}\right]=0} \\
& {\left[\mathcal{L}_{1}, Q_{a}\right]=0, \quad\left[\mathcal{L}_{1}, S_{a}\right]=0,} \\
& {\left[\mathcal{L}_{2}, Q_{a}\right]=2 i\left(\mathcal{H}-\Delta^{2}\right) S_{a}+2 \Delta \cdot \mathcal{H} Q_{a}, \quad\left[\mathcal{L}_{2}, S_{a}\right]=-2 i \mathcal{H}^{2} Q_{a}-2 \Delta \cdot \mathcal{H} S_{a}}
\end{aligned}
$$

In the limit $\alpha_{1} \rightarrow \infty$, the system (4.11) transforms into the system (4.1), and all the integrals of (4.11) are transformed into the corresponding integrals of motion of the system (4.1) with parameter $\alpha$ changed for $\alpha_{2}$. The exotic superalgebra of the system (4.11) in this limit takes the form of the exotic superalbegra of the system (4.1).

In accordance with distinct exotic nonlinear superalgebraic structures of the supersymmetric systems (4.1) and (4.11), their spectral properties also essentially differ. Consider first the system (4.1). Its integrals $\mathcal{H}, \mathcal{L}_{a}, Q_{2}$ and $S_{1}$ are $\mathcal{P} \mathcal{T}$-even operators, while $Q_{1}$ and $S_{2}$ are $\mathcal{P} \mathcal{T}$-odd :

$$
P T \mathcal{E}=\mathcal{E} P T, \quad \mathcal{E}=\mathcal{H}, \mathcal{L}_{a}, Q_{2}, S_{1} ; \quad P T \mathcal{O}=-\mathcal{O} P T, \quad \mathcal{O}=Q_{1}, S_{2}
$$

For fermionic integrals of this system the following operator identities are valid coherently with (4.6): $Q_{1} S_{1}=-S_{1} Q_{1}=Q_{2} S_{2}=-S_{2} Q_{2}=i \mathcal{L}_{2}, Q_{1} S_{2}=S_{2} Q_{1}=-Q_{2} S_{1}=-S_{1} Q_{2}=$ $\mathcal{L}_{1}$. The first order supercharges $Q_{1}$ and $Q_{2}$ commute, respectively, with the second order supercharges $S_{2}$ and $S_{1}$, and the corresponding pairs of the fermionic integrals can be diagonalized simultaneously. The functions

$$
\Psi_{k}^{ \pm}(x)=\left(\begin{array}{c}
D_{1} e^{i k x} \\
\mp i k e^{i k x}
\end{array}\right), \quad-\infty<k<\infty,
$$

are the common eigenstates of $\mathcal{H}, \mathcal{L}_{1}, Q_{2}$ and $S_{1}$ :

$$
\mathcal{H} \Psi_{k}^{ \pm}=k^{2} \Psi_{k}^{ \pm}, \quad \mathcal{L}_{1} \Psi_{k}^{ \pm}=k^{3} \Psi_{k}^{ \pm}, \quad Q_{2} \Psi_{k}^{ \pm}= \pm k \Psi_{k}^{ \pm}, \quad S_{1} \Psi_{k}^{ \pm}=\mp k^{2} \Psi_{k}^{ \pm} .
$$

The integral $\mathcal{L}_{1}$ distinguishes the eigenstates with positive and negative values of $k$, while both supercharges $Q_{2}$ and $S_{1}$ separate the eigenstates with different values + and - of the upper index. On the other hand, $Q_{1} \Psi_{k}^{ \pm}= \pm k\left(i \sigma_{3} \Psi_{k}^{ \pm}\right), S_{2} \Psi_{k}^{ \pm}= \pm k^{2}\left(i \sigma_{3} \Psi_{k}^{ \pm}\right), \mathcal{L}_{2} \Psi_{k}^{ \pm}=$ $k^{3}\left(\sigma_{3} \Psi_{k}^{ \pm}\right)$. The state with $k=0, \Psi_{0}(x)=\left(D_{1} 1,0\right)^{t}=\left(-\xi^{-1}, 0\right)^{t}$, where $t$ means a transposition, is a unique eigenstate of zero energy that is annihilated by all the integrals of motion. The $\mathcal{P} \mathcal{T}$-symmetric system (4.1) corresponds therefore to the case of unbroken exotic nonlinear supersymmetry. The commuting $\mathcal{P} \mathcal{T}$-odd supercharges $Q_{1}$ and $S_{2}$ have the common eigenfunctions $\widetilde{\Psi}_{k}^{ \pm}(x)=\left(D_{1} e^{i k x}, \pm k e^{i k x}\right)^{t}$ with real eigenvalues, $Q_{1} \widetilde{\Psi}_{k}^{ \pm}= \pm k \widetilde{\Psi}_{k}^{ \pm}$, $S_{2} \widetilde{\Psi}_{k}^{ \pm}= \pm k^{2} \widetilde{\Psi}_{k}^{ \pm}$. Analogously to the states (4.32), they are eigenfunctions of $\mathcal{H}$ and $\mathcal{L}_{1}$ with eigenvalues $k^{2}$ and $k^{3}$, respectively, and satisfy the relations $Q_{2} \widetilde{\Psi}_{k}^{ \pm}= \pm k\left(i \sigma_{3} \widetilde{\Psi}_{k}^{ \pm}\right)$, $S_{1} \widetilde{\Psi}_{k}^{ \pm}=\mp k^{2}\left(i \sigma_{3} \widetilde{\Psi}_{k}^{ \pm}\right), \mathcal{L}_{2} \widetilde{\Psi}_{k}^{ \pm}=k^{3}\left(\sigma_{3} \widetilde{\Psi}_{k}^{ \pm}\right)$. 
Consider now the system (4.11). Its integrals satisfy the same parity properties (4.31) with respect to permutations with the operator $P T$. Here we have the identities $Q_{1} S_{1}=Q_{2} S_{2}=i\left(\sigma_{3} \mathcal{L}_{1}+\Delta \mathcal{H}\right), S_{1} Q_{1}=S_{2} Q_{2}=i\left(-\sigma_{3} \mathcal{L}_{1}+\Delta \mathcal{H}\right)$, $Q_{1} S_{2}=-Q_{2} S_{1}=\mathcal{L}_{1}+\Delta \sigma_{3} \mathcal{H}, S_{2} Q_{1}=-S_{1} Q_{2}=\mathcal{L}_{1}-\Delta \sigma_{3} \mathcal{H}$. Unlike (4.1), in the system (4.11) there are no mutually commuting pairs of the first and second order supercharges, and the complete sets of mutually commuting integrals are formed by the sets of the bosonic operators $\mathcal{H}$ and $\mathcal{L}_{1}$ supplemented by one of the fermionic first order supercharges $Q_{a}$, or by one of the second order supercharges $S_{a}$.

The common eigenfunctions of the set of the $\mathcal{P} \mathcal{T}$-even operators $\left(\mathcal{H}, \mathcal{L}_{1}, S_{1}\right)$ are

$$
\Psi_{k^{2}}^{ \pm}=\left(\begin{array}{c}
D_{\alpha_{2}} e^{i k x} \\
\pm D_{\alpha_{1}} e^{i k x}
\end{array}\right), \quad-\infty<k<\infty
$$

for which $S_{1} \Psi_{k^{2}}^{ \pm}= \pm k^{2} \Psi_{k^{2}}^{ \pm}, \mathcal{H} \Psi_{k^{2}}^{ \pm}=k^{2} \Psi_{k^{2}}^{ \pm}, \mathcal{L}_{1} \Psi_{k^{2}}^{ \pm}=k^{3} \Psi_{k^{2}}^{ \pm}$. They also satisfy the relations $Q_{1} \Psi_{k^{2}}^{ \pm}= \pm i\left(k \sigma_{3}+\Delta\right) \Psi_{k^{2}}^{ \pm}, Q_{2} \Psi_{k^{2}}^{ \pm}=\mp\left(k+\sigma_{3} \Delta\right) \Psi_{k^{2}}^{ \pm}, S_{2} \Psi_{k^{2}}^{ \pm}= \pm k^{2}\left(i \sigma_{3} \Psi_{k^{2}}^{ \pm}\right), \mathcal{L}_{2} \Psi_{k^{2}}^{ \pm}=$ $k^{3}\left(\sigma_{3} \Psi_{k^{2}}^{ \pm}\right)$. The doublet of states $\Psi_{0}^{ \pm}=\left(D_{\alpha_{2}} 1, \pm D_{\alpha_{1}} 1\right)^{t}=\left(-\xi_{2}^{-1}, \mp \xi_{1}^{-1}\right)^{t}$ corresponding to $k=0$ are the eigenfunctions with the lowest, zero value of energy, $\mathcal{H} \Psi_{0}^{ \pm}=0$, which also are annihilated by both bosonic integrals $\mathcal{L}_{a}$ and both second order supercharges $S_{a}$. However, they are not annihilated by the first order supercharges, being eigenfuctions of the $Q_{1}$ with imaginary eigenvalues: $Q_{1} \Psi_{0}^{ \pm}= \pm i \Delta \Psi_{0}^{ \pm}$. Their linear combinations $\Psi_{0}^{+} \pm$ $i \Psi_{0}^{-}$are eigenfunctions of the $\mathcal{P} \mathcal{T}$-even first order supercharge $Q_{2}$ with the same pure imaginary eigenvalues $\pm i \Delta$. Unlike (4.1), the $\mathcal{P} \mathcal{T}$-symmetric system (4.11) realizes the case of spontaneously partially broken exotic nonlinear supersymmetry. Analogously, the states $\widetilde{\Psi}_{k^{2}}^{ \pm}=\left(D_{\alpha_{2}} e^{i k x}, \pm i D_{\alpha_{1}} e^{i k x}\right)^{t}$ are common eigenstates of $\mathcal{H}, \mathcal{L}_{1}$ and $S_{2}, S_{2} \widetilde{\Psi}_{k^{2}}^{ \pm}= \pm k^{2} \widetilde{\Psi}_{k^{2}}^{ \pm}$, $\mathcal{H} \widetilde{\Psi}_{k^{2}}^{ \pm}=k^{2} \widetilde{\Psi}_{k^{2}}^{ \pm}, \mathcal{L}_{1} \widetilde{\Psi}_{k^{2}}^{ \pm}=k^{3} \widetilde{\Psi}_{k^{2}}^{ \pm}$, for which we also have the relations $\mathcal{L}_{2} \widetilde{\Psi}_{k^{2}}^{ \pm}=k^{3}\left(\sigma_{3} \widetilde{\Psi}_{k^{2}}^{ \pm}\right)$, $S_{1} \widetilde{\Psi}_{k^{2}}^{ \pm}=-\mp k^{2}\left(i \sigma_{3} \widetilde{\Psi}_{k^{2}}^{ \pm}\right), Q_{1} \widetilde{\Psi}_{k^{2}}^{ \pm}=\mp\left(k+\Delta \sigma_{3}\right) \widetilde{\Psi}_{k^{2}}^{ \pm}, Q_{2} \widetilde{\Psi}_{k^{2}}^{ \pm}=\mp i\left(k \sigma_{3}+\Delta\right) \widetilde{\Psi}_{k^{2}}^{ \pm}$. The states $\widetilde{\Psi}_{0}^{ \pm}$are annihilated by $S_{a}, \mathcal{H}$ and $\mathcal{L}_{a}$, but they are eigenfunctions of $Q_{2}$ of nonzero, pure imaginary eigenvalues $\mp i \Delta$, and their linear combinations $\widetilde{\Psi}_{0}^{+} \pm i \widetilde{\Psi}_{0}^{-}$are eigenstates of $Q_{1}$ of eigenvalues $\pm i \Delta$.

According to the first relation from (4.27), the eigenvalues of the first order supercharges $Q_{a}$ have to be pure imaginary for $0 \leq k^{2}<\Delta^{2}$, equal to zero for $k^{2}=\Delta^{2}$, and to be real for energy eigenvalues $k^{2}>\Delta^{2}$. In accordance with this, the eigenstates of $Q_{1}$ with eigenvalues $\lambda= \pm i \sqrt{\Delta^{2}-k^{2}}$ are $\Psi_{k}^{ \pm}(x)=\left(\sqrt{\Delta+k} D_{\alpha_{2}} e^{i k x}, \mp \sqrt{\Delta-k} D_{\alpha_{1}} e^{i k x}\right)^{t}$ for $0 \leq k^{2}<\Delta^{2}$. Particularly, the states $\Psi_{0}^{ \pm}=\left(D_{\alpha_{2}} 1, \pm D_{\alpha_{2}} 1\right)^{t}$ are two eigenstates of eigenvalues $\pm i \Delta$ of zero energy that already have been described above. For $k^{2}>\Delta^{2}$, the eigenstates of $Q_{1}$ of real eigenvalues $\lambda= \pm \sqrt{k^{2}-\Delta^{2}}$ have the form $\Psi_{k}^{ \pm}(x)=\left(\sqrt{|\Delta+k|} D_{\alpha_{2}} e^{i k x}, \mp i \varepsilon_{k} \sqrt{|k-\Delta|} D_{\alpha_{1}} e^{i k x}\right)^{t}$, where $\varepsilon_{k}$ denotes the sign of $k$. These states $\Psi_{k}^{ \pm}(x)$ also are eigenstates of $\mathcal{H}$ and $\mathcal{L}_{1}$ of eigenvalues $k^{2}$ and $k^{3}$, respectively. The eigenstates of the $\mathcal{P} \mathcal{T}$-even supercharge $Q_{2}$ can be obtained from the eigenstates of $Q_{1}$. Denoting by $\Psi_{\lambda}$ an eigenstate of $Q_{1}$ of eigenvalue $\lambda, Q_{1} \Psi_{\lambda}=\lambda \Psi_{\lambda}$, whose explicit structure for different values of $\lambda$ we have just described, the eigenstate of $Q_{2}$ of eigenvalue $-\lambda$ is given by $\widetilde{\Psi}_{-\lambda}=\left(\Pi_{+}+i \Pi_{-}\right) \Psi_{\lambda}, Q_{2} \widetilde{\Psi}_{-\lambda}=-\lambda \widetilde{\Psi}_{-\lambda}$. Here $\Pi_{ \pm}=\frac{1}{2}\left(1 \pm \sigma_{3}\right)$ are the projectors. 
In the limit $\alpha_{1} \rightarrow \infty$, we have $\Delta \rightarrow 0$, and the described phase of the partially broken exotic nonlinear supersymmetry of the system (4.11) transmutes into the phase of unbroken exotic nonlinear supersymmetry of the system (4.1).

\section{$5 \quad$ Summary, discussion and outllok}

We investigated a special class of $\mathcal{P} \mathcal{T}$-symmetric quantum mechanical systems. Being generated via $\mathcal{P} \mathcal{T}$-regularized Darboux-Crum transformations of the free particle system $H_{0}$, each of them has the same spectrum as $H_{0}$ but with a bound state $\psi_{0,0}(x)=1$ of zero energy of $H_{0}$ transformed into a bounded (quadratically integrable on $\mathbb{R}^{1}$ ) eigenstate of the same energy of the generated system. Each such a system satisfies a stationary equation of the KdV hierarchy and is characterized by a Lax-Novikov integral being a higher order differential operator. It is a Darboux-dressed free particle momentum integral which distinguishes the left- and right-moving scattering states inside the spectral doublets and detects the bounded state of zero energy by annihilating it. Other states from the kernel of this integral are the Jordan states of the corresponding system. The peculiarity of such systems is that in them not only the reflection coefficient is zero, and so, their transmission coefficient is a pure phase, but that this phase is equal to one like for the free particle. We identify this class of the quantum systems as perfectly invisible $\mathcal{P} \mathcal{T}$-symmetric zero-gap systems. Their family includes as particular cases the $\mathcal{P} \mathcal{T}$-regularized two-particle Calogero systems (conformal quantum mechanics models of de Alfaro-Fubini-Furlan) with coupling constant values $g=n(n+1)$. The generation of the $\mathcal{P} \mathcal{T}$-symmetric zero-gap systems more complicated than the simplest case of the $\mathcal{P} \mathcal{T}$-regularized Calogero model with $n=1$ includes Jordan states of the free particle taken as the seed states in the Darboux-Crum construction.

In some systems (different from the Calogero case) we observed an interesting phenomenon of the mutual transmutation of physical and non-physical eigenstates of zero energy in the limit when one of the imaginary parameters is sent to infinity. The rational potentials of perfectly invisible $\mathcal{P} \mathcal{T}$-symmetric zero-gap systems, being solutions of stationary equations of the KdV hierarchy, can be promoted to the solutions of time-dependent equations from the same hierarchy. The interesting peculiarity of time-dependent solutions we constructed is that under appropriate choice of the parameters they reveal a behaviour being typical for extreme waves. It is worth noting here that if we separate a $\mathcal{P} \mathcal{T}$-symmetric function $u(x, t)$ in real and imaginary parts, $u(x, t)=v(x, t)+i w(x, t)$, in the case of the $\mathrm{KdV}$ equation we obtain an equivalent system of two coupled equations

$$
v_{t}-3\left(v^{2}-w^{2}\right)_{x}+v_{x x x}=0, \quad w_{t}-6(v w)_{x}+w_{x x x}=0
$$

for two real fields $v(x, t)$ and $w(x, t)$ instead of one equation for the complex-valued field $u(x, t)$. At $w=0$, this system reduces to the KdV equation, while for $v=0$ it corresponds to the case of the linearized $\mathrm{KdV}$ equation. We refer here to $[63,64]$ where different aspects of the system (5.1) were discussed in the context of the complex KdV equation.

We showed that the two simplest Hamiltonians from the Calogero subfamily with $n=1$ and $n=2$ governing the dynamics in conformal invariant $\mathcal{P} \mathcal{T}$-symmetric quantum mechanical systems also determine the fluctuation spectra around the singular kinks arising 
as traveling waves in the field-theoretical Liouville and SU(3) conformal Toda systems. By pushing the centers of the kinks to the imaginary axis we regularize them and the operators governing the fluctuations around them take the form of the Hamiltonians of the corresponding $\mathcal{P} \mathcal{T}$-regularized Calogero systems. This picture can be related to the contraction of the infinite-dimensional conformal group in the two-dimensional Minkowskian setting to a finite subgroup in conformal quantum mechanics.

The peculiar properties of the investigated class of the systems are reflected in the quantum mechanical supersymmetry associated with them. In the extended systems composed from the pairs of such systems related by a first order Darboux transformation, the conventional $\mathcal{N}=2$ supersymmetry is extended to exotic $\mathcal{N}=4$ nonlinear supersymmetry. The latter includes an additional pair of supercharges to be matrix differential operators of the even order constructed from the higher order differential operators which intertwine the Hamiltonians of the subsystems via a 'virtual' free particle system. As a result, the first and higher order supercharges generate the Lax-Novikov integrals of the subsystems which compose one of the additional bosonic integrals of the extended system being a central charge $\mathcal{L}_{1}$ of the superalgebra alongside with the Hamiltonian operator $\mathcal{H}$. Another additional bosonic integral corresponds to the operator $\mathcal{L}_{2}=\sigma_{3} \mathcal{L}_{1}$ that generates a rotation between the first and higher order supercharges with coefficients to be powers of the Hamiltonian. The appearance of the Hamiltonian in the structure coefficients of the superalgebra corresponds to a nonlinear nature of the exotic supersymmetry of such systems. This described family of the extended systems realizes the case of the unbroken exotic supersymmetry in which there is one singlet state of zero eigenvalue of $\mathcal{H}$ which is annihilated by all the four supercharges and by both additional bosonic integrals. The system of the $\mathcal{P} \mathcal{T}$-regularized kinks of the $\mathrm{SU}(3)$ conformal Toda system can be described by such unbroken exotic $\mathcal{N}=4$ nonlinear supersymmetry. There is also another family of the extended two-component systems corresponding to the case of the partially broken exotic supersymmetry in which a doublet of zero energy states is annihilated by the bosonic Lax integrals and higher-order supercharges but is not annihilated by the first order supercharges. We considered a simplest example of such a supersymmetric system in which the first order supercharges are composed from the first order operators which intertwine the completely isospectral subsystems directly, while the intertwining operators in the structure of the pair of the second order supercharges relate the subsystems via a virtual free particle system. In a certain limit such a system with partially broken exotic supersymmetry transmutes into the perfectly invisible extended system described by unbroken supersymmetry.

Having in mind the analogy with the case of reflectionless quantum systems related to the conventional multi-soliton solutions of the $\mathrm{KdV}$ [57], one can expect that there exists an infinite chain of pairs of the perfectly invisible $\mathcal{P} \mathcal{T}$-symmetric zero-gap systems in which one of the neighbour pairs is described by unbroken exotic supersymmetry while another neighbour pair is characterized by partially broken phase of the exotic nonlinear supersymmetry and the neighbour pairs with different phases of supersymmetry can be transmuted via the process of soliton scattering. It would be interesting to construct the indicated infinite chain of the pairs of the perfectly invisible $\mathcal{P} \mathcal{T}$-symmetric zero-gap Schrödinger systems. One of the first order supercharges in such chains of the systems can 
then be considered as a Hamiltonian of a $(1+1)$-dimensional Dirac system with a perfectly invisible $\mathcal{P} \mathcal{T}$-symmetric scalar potential that descirbes a fermion in a multi-kink-antikink background [58].

The $\mathcal{P} \mathcal{T}$-regularized perfectly invisible two-particle Calogero systems we considered possess conformal symmetry like their conventional counterparts with real inverse square potentials [29]. The interesting question is what happens with conformal symmetry in the perfectly invisible $\mathcal{P} \mathcal{T}$-symmetric zero-gap systems of different form, like in the systems described by the $\mathcal{P} \mathcal{T}$-symmetric potentials (2.33) and (2.39). Another, related question is what happens with the exotic nonlinear supersymmetry we considered under extension of it by taking into account the conformal symmetry. A priori it is not clear whether in such a case we obtain some finite nonlineaer superalgebraic structure or some infinite-dimensional superalgebraic structure will be generated, cf. [59-61]. We are going to consider these questions elsewhere [62].

The interesting question also is whether perfectly invisible $\mathcal{P} \mathcal{T}$-symmetric zero-gap systems to be more complicated than the $\mathcal{P} \mathcal{T}$-regularized Calogero systems with $n=1$ and $n=2$ can be related to the field-theoretical conformal models.

In conclusion we note that in [25] there were considered self-similar solutions of the $\mathrm{KdV}$ equation with initial conditions of the form $N(N+1) / x^{2}$, which are rational singular solutions on the real line. Following [26], one could consider the solutions to the Schrödinger problem with such a potential on half-line $x>0$ (see appendix) and then formally extend the same solutions to another side $x<0$ of the pole as it is done in [26] for the so called positon solutions. With such a formal treatment of solutions one could arrive at the conclusion that the indicated rational potentials are reflectionless on all the real line, and moreover, that they would be characterized by the transmission amplitude equal to one. Treated in this way singular potentials are called in [26] as "super-transparent" or "super-reflectionless". However, physically such a treatment of singular potentials on all the real line is completely formal since the second order pole singularity at $x=0$, unlike the case of delta function potential, is not penetrable, and the physically admissible states in the regions $x<0$ and $x>0$ do not mix dynamically. The impermissibility of such treatment of the quantum problem also is reflected by the mentioned in section 1 observation that the Lax-Novikov operator commuting with Hamiltonian in this case is not observable since it transforms physical states into non-physical ones [28]. It is the $\mathcal{P} \mathcal{T}$-symmetric regularization considered here that radically changes the properties of such systems and their interpretation. It would be interesting to apply the $\mathcal{P} \mathcal{T}$-symmetric regularization to the generalized Darboux transformations used in [26] for the construction of time-dependent positon and soliton-positon solutions of the KdV equation.

\section{Acknowledgments}

We acknowledge support from research projects FONDECYT 1130017 and Convenio Marco Universidades del Estado (Project USA1555), Chile, and MINECO (Project MTM201457129-C2-1-P), Spain. JMG also acknowledges the Junta de Castilla y León for financial 
support under grant VA057U16. MSP is grateful for the warm hospitality at Salamanca and Valladolid Universities where a part of this work was done.

\section{A Quantum scattering on the half-line}

Here we briefly discuss a general picture of the quantum scattering problem on the half-line.

Consider a free particle of mass $m=\frac{1}{2}$ on the half-line $(0, \infty)$ given by the Hamiltonian operator

$$
H=-\frac{d^{2}}{d x^{2}}+U(x)
$$

with $U(x)=0$ for $x>0$ and $U(x)=+\infty$ for $x \leq 0$. Its bounded eigenstates of energy $E=k^{2}>0$ with $k>0$ are described by wave functions $\psi_{k}(x)=C \sin k x$ for $x>0$ and $\psi_{k}(x)=0$ for $x \leq 0$. They represent a linear combination of two plane waves, $\psi_{k}(x)=e^{-i k x}-e^{i k x}$, in which the first term can be interpreted as an incident from $x=+\infty$ plane wave, while the second term can be considered as a reflected wave.

Let us consider now the case of a quantum particle on the half-line $(0, \infty)$ subject to a nontrivial potential $U(x)$ that disappears sufficiently rapidly at $x=+\infty$, and $U(x) \rightarrow+\infty$ when $x \rightarrow 0$ in such a way that the particle cannot penetrate to the negative half-line: $\psi(0)=0$ for $x \leq 0$. Then for eigenfunction corresponding to eigenvalue $E=k^{2}>0$ the probability current $j(x) \propto \psi_{k}^{*}(x)\left(\psi_{k}(x)\right)^{\prime}-\left(\psi_{k}^{*}(x)\right)^{\prime} \psi_{k}(x)$ disappears when $x \rightarrow 0^{+}$: $j\left(0^{+}\right)=0$. For $x \rightarrow+\infty$, we fix the asymptotic form of the solution of the equation $H \psi_{k}(x)=k^{2} \psi_{k}(x)$ in a form

$$
\psi_{k}(x)=e^{-i k x}+r e^{i k x},
$$

where $r$ is a complex constant that can be interpreted as a reflection amplitude. The continuity equation $\frac{\partial}{\partial t} \rho+\frac{\partial}{\partial x} j=0$ for stationary solutions of the corresponding time-dependent Schrödinger equation reduces to the condition $\frac{\partial}{\partial x} j=0$. Since $j\left(0^{+}\right)=0$, the probability current $j(x)=0$ for any $x \in(0, \infty)$. For asymptotic form of the solution (A.2) this gives $r r^{*}=1$, i.e. the reflection amplitude $r$ is a pure phase. In the case of the free paticle on the half-line we have $r=-1$. In the case of the potential $U(x)=n(n+1) / x^{2}$, the solutions are given by the Darboux-Crum mapping of the free particle solutions: $\psi_{k}^{(n)}(x)=\mathcal{D}_{n} \sin k x$, where $\mathcal{D}_{n}=D_{n} \ldots D_{1}, D_{j}=\frac{d}{d x}-\frac{j}{x}, j=1, \ldots, n$. These eigenfunctions $\psi_{k}^{(n)}(x)$ and their derivatives up to (including) the order $n$ annul at $x=0$. For $x \rightarrow+\infty$, the asymptotic form of eigenfunctions is $\psi_{k}^{(n)}(x)=-(-i k)^{n}\left(e^{-i k x}+(-1)^{n+1} e^{i k x}\right)$. From here we find that the system with potential $U(x)=n(n+1) / x^{2}$ is charcterized by reflection amplitude $r=$ $(-1)^{n+1}$. Thus the cases with even $n$ are characterized by the same reflection amplitude $r=$ -1 as the free particle, while for odd $n$ we have $r=+1$. By this reason if (A.1) with $U(x)=$ $n(n+1) / x^{2}$ is considered as a Hamiltonian operator in relative coordinate for a two-particle system, the cases with even and odd $n$ correspond to bosons and fermions, respectively.

This picture can be generalized to the anyonic case $[65,66]$ by considering the same potential but with coupling constant changed for $g=\nu(\nu+1)$ with $\nu>0$. The general solution to the stationary Schrödinger equation for eigenvalue $E=k^{2}>0$ with $k>0$ is $\psi_{k}^{(\nu)}(x)=\sqrt{x}\left(C_{1} J_{\beta}(k x)+C_{2} Y_{\beta}(k x)\right)$, where $\beta=\nu+\frac{1}{2}$ and $J_{\beta}$ and $Y_{\beta}$ are Bessel functions of the first and second kind, respectively. The boundary condition at $x=0^{+}$ 
fixes $C_{2}=0$, and from asymptotic form of the Bessel function for $x \rightarrow+\infty, J_{\beta}(x)=$ $\sqrt{\frac{2}{\pi x}} \cos \left(x-\frac{\beta \pi}{2}-\frac{\pi}{4}\right)$, we find that in this case $r=\exp [-i \pi(\nu+1)]$, that generalizes the bosonic and fermionic cases considered above for the anyonic case of arbitrary $\nu>0$.

Open Access. This article is distributed under the terms of the Creative Commons Attribution License (CC-BY 4.0), which permits any use, distribution and reproduction in any medium, provided the original author(s) and source are credited.

\section{References}

[1] C.M. Bender and S. Boettcher, Real spectra in non-Hermitian Hamiltonians having PT symmetry, Phys. Rev. Lett. 80 (1998) 5243 [physics/9712001] [INSPIRE].

[2] C.M. Bender, Making sense of non-Hermitian Hamiltonians, Rept. Prog. Phys. 70 (2007) 947 [hep-th/0703096] [InSPIRE].

[3] A. Mostafazadeh, Pseudo-Hermitian representation of quantum mechanics, Int. J. Geom. Meth. Mod. Phys. 7 (2010) 1191 [arXiv:0810.5643] [InSPIRE].

[4] P. Dorey, C. Dunning and R. Tateo, Spectral equivalences, Bethe ansatz equations and reality properties in PT-symmetric quantum mechanics, J. Phys. A 34 (2001) 5679 [hep-th/0103051] [INSPIRE].

[5] P. Dorey, C. Dunning and R. Tateo, The ODE/IM correspondence, J. Phys. A 40 (2007) R205 [hep-th/0703066] [inSPIRE].

[6] F. Calogero, Solution of the one-dimensional $N$ body problems with quadratic and/or inversely quadratic pair potentials, J. Math. Phys. 12 (1971) 419 [InSPIRE].

[7] M. Znojil and M. Tater, Complex Calogero model with real energies, J. Phys. A 34 (2001) 1793 [quant-ph/0010087].

[8] P.K. Ghosh and K.S. Gupta, On the real spectra of Calogero model with complex coupling, Phys. Lett. A 323 (2004) 29 [hep-th/0310276] [INSPIRE].

[9] B. Basu-Mallick, T. Bhattacharyya, A. Kundu and B.P. Mandal, Bound and scattering states of extended Calogero model with an additional PT invariant interaction, Czech. J. Phys. 54 (2004) 5 [hep-th/0309136] [InSPIRE].

[10] A. Fring and M. Znojil, PT-symmetric deformations of Calogero models, J. Phys. A 41 (2008) 194010 [arXiv:0802.0624] [InSPIRE].

[11] A. Fring and M. Smith, Non-Hermitian multi-particle systems from complex root spaces, J. Phys. A 45 (2012) 085203 [arXiv:1108.1719] [INSPIRE].

[12] A. Fring, PT-symmetric deformations of integrable models, Phil. Trans. Roy. Soc. Lond. A 371 (2013) 20120046 [arXiv:1204.2291] [INSPIRE].

[13] F. Correa and O. Lechtenfeld, PT deformation of angular Calogero models, JHEP 11 (2017) 122 [arXiv:1705.05425] [INSPIRE].

[14] H. Airault, H.P. McKean and J. Moser, Rational and elliptic solutions of the Korteweg-de Vries equation and a related many-body problem, Comm. Pure Appl. Math. 30 (1977) 95.

[15] M. Adler and J. Moser, On a class of polynomials connected with the Korteweg-de Vries equation, Commun. Math. Phys. 61 (1978) 1 [INSPIRE]. 
[16] A. Gorsky and N. Nekrasov, Hamiltonian systems of Calogero type and two-dimensional Yang-Mills theory, Nucl. Phys. B 414 (1994) 213 [hep-th/9304047] [INSPIRE].

[17] M. Znojil, F. Cannata, B. Bagchi and R. Roychoudhury, Supersymmetry without hermiticity within PT symmetric quantum mechanics, Phys. Lett. B 483 (2000) 284 [hep-th/0003277] [INSPIRE].

[18] M. Znojil, PT symmetrized supersymmetric quantum mechanics, Czech. J. Phys. 51 (2001) 420 [hep-ph/0101038] [INSPIRE].

[19] P. Dorey, C. Dunning and R. Tateo, Supersymmetry and the spontaneous breakdown of PT symmetry, J. Phys. A 34 (2001) L391 [hep-th/0104119] [inSPIRE].

[20] B. Bagchi, S. Mallik and C. Quesne, Complexified PSUSY and SSUSY interpretations of some PT symmetric Hamiltonians possessing two series of real energy eigenvalues, Int. J. Mod. Phys. A 17 (2002) 51 [quant-ph/0106021] [INSPIRE].

[21] F. Correa and M.S. Plyushchay, Self-isospectral tri-supersymmetry in PT-symmetric quantum systems with pure imaginary periodicity, Annals Phys. 327 (2012) 1761 [arXiv:1201.2750] [INSPIRE].

[22] F. Correa and M.S. Plyushchay, Spectral singularities in PT-symmetric periodic finite-gap systems, Phys. Rev. D 86 (2012) 085028 [arXiv:1208.4448] [INSPIRE].

[23] S.P. Novikov, S.V. Manakov, L.P. Pitaevskii and V.E. Zakharov, Theory of solitons, Plenum, New York U.S.A., (1984).

[24] V.B. Matveev and M.A. Salle, Darboux transformations and solitons, Springer, Berlin Germany, (1991).

[25] L.A. Bordag and V.B. Matveev, Self-similar solutions of the Korteweg-de Vries equation and potentials with a trivial S-matrix, Theor. Math. Phys. 34 (1978) 272 [Teor. Mat. Fiz. 34 (1978) 426].

[26] V.B. Matveev, Positons: slowly decreasing analogues of solitons, Theor. Math. Phys. 131 (2002) 483 [Teor. Mat. Fiz. 131 (2002) 44] [InSPIRE].

[27] A. Arancibia and M.S. Plyushchay, Chiral asymmetry in propagation of soliton defects in crystalline backgrounds, Phys. Rev. D 92 (2015) 105009 [arXiv:1507.07060] [INSPIRE].

[28] F. Correa, M.A. del Olmo and M.S. Plyushchay, On hidden broken nonlinear superconformal symmetry of conformal mechanics and nature of double nonlinear superconformal symmetry, Phys. Lett. B 628 (2005) 157 [hep-th/0508223] [INSPIRE].

[29] V. de Alfaro, S. Fubini and G. Furlan, Conformal invariance in quantum mechanics, Nuovo Cim. A 34 (1976) 569 [INSPIRE].

[30] C.M. Bender, D.C. Brody, J. Chen and E. Furlan, PT-symmetric extension of the Korteweg-de Vries equation, J. Phys. A 40 (2007) F153 [math-ph/0610003] [InSPIRE].

[31] A. Fring, PT-symmetric deformations of the Korteweg-de Vries equation, J. Phys. A 40 (2007) 4215 [math-ph/0701036] [INSPIRE].

[32] C.M. Bender and J. Feinberg, Does the complex deformation of the Riemann equation exhibit shocks?, J. Phys. A 41 (2008) 244004 [arXiv:0709.2727] [INSPIRE].

[33] A. Cavaglia and A. Fring, PT-symmetrically deformed shock waves, J. Phys. A 45 (2012) 444010 [arXiv:1201.5809] [InSPIRE]. 
[34] S. Longhi and G. Della Valle, Invisible defects in complex crystals, Annals Phys. 334 (2013) 35 [arXiv:1306.0667].

[35] F. Correa, V. Jakubsky and M.S. Plyushchay, PT-symmetric invisible defects and confluent Darboux-Crum transformations, Phys. Rev. A 92 (2015) 023839 [arXiv:1506.00991] [INSPIRE].

[36] V.V. Konotop, J. Yang and D.A. Zezyulin, Nonlinear waves in PT-symmetric systems, Rev. Mod. Phys. 88 (2016) 035002 [arXiv:1603.06826] [InSPIRE].

[37] J.F. Cariñena and M.S. Plyushchay, Ground-state isolation and discrete flows in a rationally extended quantum harmonic oscillator, Phys. Rev. D 94 (2016) 105022 [arXiv:1611.08051] [INSPIRE].

[38] F. Correa and M.S. Plyushchay, Hidden supersymmetry in quantum bosonic systems, Annals Phys. 322 (2007) 2493 [hep-th/0605104] [INSPIRE].

[39] J.L. Burchnall and T.W. Chaundy, Commutative ordinary differential operators, Proc. London Math. Soc. 21 (1923) 420.

[40] J.L. Burchnall and T.W. Chaundy, Commutative ordinary differential operators, Proc. Roy. Soc. London A 118 (1928) 557.

[41] I.M. Krichever, Integration of nonlinear equations by the methods of algebraic geometry, Funct. Anal. Appl. 11 (1977) 12.

[42] I.M. Krichever, Baker-Akhiezer functions and integrable systems, in Integrability. The Seiberg-Witten and Whitham equations, H.W. Braden and I.M. Krichever eds., Gordon and Breach Science Publishers, Amsterdam The Netherlands, (2000), pg. 1.

[43] A. Schulze-Halberg, Wronskian representation for confluent supersymmetric transformation chains of arbitrary order, Eur. Phys. J. Plus 128 (2013) 68.

[44] A. Contreras-Astorga and A. Schulze-Halberg, Recursive representation of Wronskians in confluent supersymmetric quantum mechanics, J. Phys. A 50 (2017) 105301 [arXiv: 1702.00843] [INSPIRE].

[45] P. Drazin and R. Johnson, Solitons: an introduction, Cambridge University Press, Cambridge U.K., (1996).

[46] J. Kumar, Conformal mechanics and the Virasoro algebra, JHEP 04 (1999) 006 [hep-th/9901139] [INSPIRE].

[47] S. Cacciatori, D. Klemm and D. Zanon, $W_{\infty}$ algebras, conformal mechanics and black holes, Class. Quant. Grav. 17 (2000) 1731 [hep-th/9910065] [INSPIRE].

[48] E. D'Hoker and R. Jackiw, Liouville field theory, Phys. Rev. D 26 (1982) 3517 [INSPIRE].

[49] R. Jackiw, Liouville field theory: a two-dimensional model for gravity?, in Quantum theory of gravity, S. Christensen ed, Adam Hilger, Bristol U.K., (1984), pg. 403.

[50] A. Bilal and J.-L. Gervais, Extended $C=\infty$ conformal systems from classical Toda field theories, Nucl. Phys. B 314 (1989) 646 [InSPIRE].

[51] K.E. Cahill, A. Comtet and R.J. Glauber, Mass formulas for static solitons, Phys. Lett. B 64 (1976) 283 [INSPIRE].

[52] J. Mateos Guilarte, A. Alonso-Izquierdo, W. Garcia Fuertes, M. de la Torre Mayado and M.J. Senosiain, Quantum fluctuations around low-dimensional topological defects, PoS (ISFTG) 013 [arXiv: 0909.2107] [INSPIRE]. 
[53] A. Alonso-Izquierdo and J. Mateos Guilarte, One-loop kink mass shifts: a computational approach, Nucl. Phys. B 852 (2011) 696 [arXiv:1107.2216] [INSPIRE].

[54] A. Arancibia, J. Mateos Guilarte and M.S. Plyushchay, Effect of scalings and translations on the supersymmetric quantum mechanical structure of soliton systems, Phys. Rev. D 87 (2013) 045009 [arXiv:1210.3666] [InSPIRE].

[55] F. Correa, V. Jakubsky, L.-M. Nieto and M.S. Plyushchay, Self-isospectrality, special supersymmetry and their effect on the band structure, Phys. Rev. Lett. 101 (2008) 030403 [arXiv:0801.1671] [INSPIRE].

[56] M.S. Plyushchay and L.-M. Nieto, Self-isospectrality, mirror symmetry and exotic nonlinear supersymmetry, Phys. Rev. D 82 (2010) 065022 [arXiv:1007.1962] [INSPIRE].

[57] A. Arancibia and M.S. Plyushchay, Transmutations of supersymmetry through soliton scattering and self-consistent condensates, Phys. Rev. D 90 (2014) 025008 [arXiv: 1401.6709] [INSPIRE].

[58] A. Arancibia, J. Mateos Guilarte and M.S. Plyushchay, Fermion in a multi-kink-antikink soliton background and exotic supersymmetry, Phys. Rev. D 88 (2013) 085034 [arXiv: 1309.1816] [INSPIRE].

[59] S. Fedoruk, E. Ivanov and O. Lechtenfeld, OSp(4|2) superconformal mechanics, JHEP 08 (2009) 081 [arXiv: 0905.4951] [INSPIRE].

[60] E. Ivanov, S. Krivonos and O. Lechtenfeld, New variant of $N=4$ superconformal mechanics, JHEP 03 (2003) 014 [hep-th/0212303] [INSPIRE].

[61] C. Leiva and M.S. Plyushchay, Superconformal mechanics and nonlinear supersymmetry, JHEP 10 (2003) 069 [hep-th/0304257] [INSPIRE].

[62] J. Mateos Guilarte and M.S. Plyushchay, Extended nonlinear super-Schrödinger symmetry of PT-symmetric perfectly invisible zero-gap quantum systems, in preparation.

[63] A. Cavaglia, A. Fring and B. Bagchi, PT-symmetry breaking in complex nonlinear wave equations and their deformations, J. Phys. A 44 (2011) 325201 [arXiv:1103.1832] [INSPIRE].

[64] J. Cen and A. Fring, Complex solitons with real energies, J. Phys. A 49 (2016) 365202 [arXiv: 1602.05465] [INSPIRE].

[65] J.M. Leinaas and J. Myrheim, On the theory of identical particles, Nuovo Cim. B 37 (1977) 1 [INSPIRE].

[66] A.P. Polychronakos, Physics and mathematics of Calogero particles, J. Phys. A 39 (2006) 12793 [hep-th/0607033] [inSPIRE]. 